\title{
Neutral strange particle production in deep inelastic scattering at HERA
}

\author{
ZEUS Collaboration
}

\begin{abstract}
This paper presents measurements of $K^{0}$ and $\Lambda$ production in neutral current, deep inelastic scattering of $26.7 \mathrm{GeV}$ electrons and $820 \mathrm{GeV}$ protons in the kinematic range $10<Q^{2}<640$ $\mathrm{GeV}^{2}, 0.0003<x<0.01$, and $y>0.04$. Average multiplicities for $K^{0}$ and $\Lambda$ production are determined for transverse momenta $p_{\mathrm{T}}>0.5 \mathrm{GeV}$ and pseudorapidities $|\eta|<1.3$. The multiplicities favour a stronger strange to light quark suppression in the fragmentation chain than found in $e^{+} e^{-}$ experiments. The production properties of $K^{0}$ s in events with and without a large rapidity gap with respect to the proton direction are compared. The ratio of neutral $K^{0}$ 's to charged particles per event in the measured kinematic range is, within the present statistics, the same in both samples.
\end{abstract}




\section{The ZEUS Collaboration}

M. Derrick, D. Krakauer, S. Magill, D. Mikunas, B. Musgrave, J. Repond, R. Stanek, R.L. Talaga, H. Zhang Argonne National Laboratory, Argonne, IL, USA ${ }^{p}$

R. Ayad ${ }^{1}$, G. Bari, M. Basile, L. Bellagamba, D. Boscherini, A. Bruni, G. Bruni, P. Bruni, G. Cara Romeo, G. Castellini ${ }^{2}$, M. Chiarini, L. Cifarelli ${ }^{3}$, F. Cindolo, A. Contin, M. Corradi, I. Gialas ${ }^{4}$, P. Giusti, G. Iacobucci, G. Laurenti, G. Levi, A. Margotti, T. Massam, R. Nania, C. Nemoz,

F. Palmonari, A. Polini, G. Sartorelli, R. Timellini, Y. Zamora Garcia ${ }^{1}$, A. Zichichi

University and INFN Bologna, Bologna, Italy ${ }^{f}$

A. Bargende ${ }^{5}$, J. Crittenden, K. Desch, B. Diekmann ${ }^{6}$, T. Doeker, M. Eckert, L. Feld, A. Frey, M. Geerts, G. Geitz ${ }^{7}$, M. Grothe, T. Haas, H. Hartmann, K. Heinloth, E. Hilger,

H.-P. Jakob, U.F. Katz, S.M. Mari ${ }^{4}$, A. Mass ${ }^{8}$, S. Mengel, J. Mollen, E. Paul, Ch. Rembser, D. Schramm, J. Stamm, R. Wedemeyer

Physikalisches Institut der Universität Bonn, Bonn, Federal Republic of Germany ${ }^{c}$

S. Campbell-Robson, A. Cassidy, N. Dyce, B. Foster, S. George, R. Gilmore, G.P. Heath, H.F. Heath, T.J. Llewellyn, C.J.S. Morgado, D.J.P. Norman, J.A. O'Mara, R.J. Tapper, S.S. Wilson, R. Yoshida

H.H. Wills Physics Laboratory, University of Bristol, Bristol, U.K. ${ }^{\circ}$

R.R. Rau

Brookhaven National Laboratory, Upton, L.I., USA ${ }^{p}$

M. Arneodo ${ }^{9}$, L. Iannotti, M. Schioppa, G. Susinno

Calabria University, Physics Dept.and INFN, Cosenza, Italy ${ }^{f}$

A. Bernstein, A. Caldwell, N. Cartiglia, J.A. Parsons, S. Ritz ${ }^{10}$, F. Sciulli, P.B. Straub, L. Wai, S. Yang, Q. Zhu

Columbia University, Nevis Labs., Irvington on Hudson, N.Y., USA ${ }^{q}$

P. Borzemski, J. Chwastowski, A. Eskreys, K. Piotrzkowski, M. Zachara, L. Zawiejski

Inst. of Nuclear Physics, Cracow, Poland ${ }^{j}$

L. Adamczyk, B. Bednarek, K. Jeleń, D. Kisielewska, T. Kowalski, E. Rulikowska-Zarȩbska,

L. Suszycki, J. Zając

Faculty of Physics and Nuclear Techniques, Academy of Mining and Metallurgy, Cracow, Poland ${ }^{j}$

A. Kotański, M. Przybycień

Jagellonian Univ., Dept. of Physics, Cracow, Poland ${ }^{k}$

L.A.T. Bauerdick, U. Behrens, H. Beier ${ }^{11}$, J.K. Bienlein, C. Coldewey, O. Deppe, K. Desler, G. Drews,

M. Flasiński ${ }^{12}$, D.J. Gilkinson, C. Glasman, P. Göttlicher, J. Große-Knetter, B. Gutjahr ${ }^{13}$, W. Hain, D. Hasell, H. Heßling, Y. Iga, P. Joos, M. Kasemann, R. Klanner, W. Koch, L. Köpke ${ }^{14}$, U. Kötz, H. Kowalski, J. Labs, A. Ladage, B. Löhr, M. Löwe, D. Lüke, J. Mainusch, O. Mańczak, T. Monteiro ${ }^{15}$, J.S.T. Ng, S. Nickel ${ }^{16}$, D. Notz, K. Ohrenberg, M. Roco, M. Rohde, J. Roldán, U. Schneekloth, W. Schulz, F. Selonke, E. Stiliaris ${ }^{17}$, B. Surrow, T. Voß, D. Westphal, G. Wolf, C. Youngman, J.F. Zhou

Deutsches Elektronen-Synchrotron DESY, Hamburg, Federal Republic of Germany

H.J. Grabosch, A. Kharchilava, A. Leich, M.C.K. Mattingly, A. Meyer, S. Schlenstedt, N. Wulff

DESY-Zeuthen, Inst. für Hochenergiephysik, Zeuthen, Federal Republic of Germany

G. Barbagli, P. Pelfer

University and INFN, Florence, Italy ${ }^{f}$

G. Anzivino, G. Maccarrone, S. De Pasquale, L. Votano

INFN, Laboratori Nazionali di Frascati, Frascati, Italy ${ }^{f}$

A. Bamberger, S. Eisenhardt, A. Freidhof, S. Söldner-Rembold ${ }^{18}$, J. Schroeder ${ }^{19}$, T. Trefzger

Fakultät für Physik der Universität Freiburg i.Br., Freiburg i.Br., Federal Republic of Germany ${ }^{c}$ 
N.H. Brook, P.J. Bussey, A.T. Doyle ${ }^{20}$, J.I. Fleck ${ }^{4}$, D.H. Saxon, M.L. Utley, A.S. Wilson Dept. of Physics and Astronomy, University of Glasgow, Glasgow, U.K. ${ }^{\circ}$

A. Dannemann, U. Holm, D. Horstmann, T. Neumann, R. Sinkus, K. Wick

Hamburg University, I. Institute of Exp. Physics, Hamburg, Federal Republic of Germany ${ }^{c}$

E. Badura ${ }^{21}$, B.D. Burow ${ }^{22}$, L. Hagge, E. Lohrmann, J. Milewski, M. Nakahata ${ }^{23}$, N. Pavel, G. Poelz, W. Schott, F. Zetsche

Hamburg University, II. Institute of Exp. Physics, Hamburg, Federal Republic of Germany ${ }^{c}$

T.C. Bacon, I. Butterworth, E. Gallo, V.L. Harris, B.Y.H. Hung, K.R. Long, D.B. Miller, P.P.O. Morawitz, A. Prinias, J.K. Sedgbeer, A.F. Whitfield

Imperial College London, High Energy Nuclear Physics Group, London, U.K. ${ }^{o}$

U. Mallik, E. McCliment, M.Z. Wang, S.M. Wang, J.T. Wu, Y. Zhang

University of Iowa, Physics and Astronomy Dept., Iowa City, USA ${ }^{p}$

P. Cloth, D. Filges

Forschungszentrum Jülich, Institut für Kernphysik, Jülich, Federal Republic of Germany

S.H. An, S.M. Hong, S.W. Nam, S.K. Park, M.H. Suh, S.H. Yon

Korea University, Seoul, Korea ${ }^{h}$

R. Imlay, S. Kartik, H.-J. Kim, R.R. McNeil, W. Metcalf, V.K. Nadendla

Louisiana State University, Dept. of Physics and Astronomy, Baton Rouge, LA, USA ${ }^{p}$

F. Barreiro ${ }^{24}$, G. Cases, J.P. Fernandez, R. Graciani, J.M. Hernández, L. Hervás ${ }^{24}$, L. Labarga ${ }^{24}$, M. Martinez, J. del Peso, J. Puga, J. Terron, J.F. de Trocóniz

Univer. Autónoma Madrid, Depto de Física Teóríca, Madrid, Spain ${ }^{n}$

G.R. Smith

University of Manitoba, Dept. of Physics, Winnipeg, Manitoba, Canada ${ }^{a}$

F. Corriveau, D.S. Hanna, J. Hartmann, L.W. Hung, J.N. Lim, C.G. Matthews, P.M. Patel,

L.E. Sinclair, D.G. Stairs, M. St.Laurent, R. Ullmann, G. Zacek

McGill University, Dept. of Physics, Montréal, Québec, Canada ${ }^{a, ~} b$

V. Bashkirov, B.A. Dolgoshein, A. Stifutkin

Moscow Engineering Physics Institute, Mosocw, Russia ${ }^{l}$

G.L. Bashindzhagyan, P.F. Ermolov, L.K. Gladilin, Y.A. Golubkov, V.D. Kobrin, V.A. Kuzmin, A.S. Proskuryakov, A.A. Savin, L.M. Shcheglova, A.N. Solomin, N.P. Zotov

Moscow State University, Institute of Nuclear Physics, Moscow, Russia ${ }^{m}$

M. Botje, F. Chlebana, A. Dake, J. Engelen, M. de Kamps, P. Kooijman, A. Kruse, H. Tiecke, W. Verkerke, M. Vreeswijk, L. Wiggers, E. de Wolf, R. van Woudenberg NIKHEF and University of Amsterdam, Netherlands ${ }^{i}$

D. Acosta, B. Bylsma, L.S. Durkin, K. Honscheid, C. Li, T.Y. Ling, K.W. McLean ${ }^{25}$, W.N. Murray, I.H. Park, T.A. Romanowski ${ }^{26}, \mathrm{R}$. Seidlein ${ }^{27}$

Ohio State University, Physics Department, Columbus, Ohio, USA ${ }^{p}$

D.S. Bailey, A. Byrne ${ }^{28}$, R.J. Cashmore, A.M. Cooper-Sarkar, R.C.E. Devenish, N. Harnew,

M. Lancaster, L. Lindemann ${ }^{4}$, J.D. McFall, C. Nath, V.A. Noyes, A. Quadt, J.R. Tickner,

H. Uijterwaal, R. Walczak, D.S. Waters, F.F. Wilson, T. Yip

Department of Physics, University of Oxford, Oxford, U.K. ${ }^{\circ}$

G. Abbiendi, A. Bertolin, R. Brugnera, R. Carlin, F. Dal Corso, M. De Giorgi, U. Dosselli, S. Limentani, M. Morandin, M. Posocco, L. Stanco, R. Stroili, C. Voci

Dipartimento di Fisica dell' Universita and INFN, Padova, Italy ${ }^{f}$ 
J. Bulmahn, J.M. Butterworth, R.G. Feild, B.Y. Oh, J.J. Whitmore ${ }^{29}$

Pennsylvania State University, Dept. of Physics, University Park, PA, USA ${ }^{q}$

G. D’Agostini, G. Marini, A. Nigro, E. Tassi

Dipartimento di Fisica, Univ. 'La Sapienza' and INFN, Rome, Italy ${ }^{f}$

J.C. Hart, N.A. McCubbin, K. Prytz, T.P. Shah, T.L. Short

Rutherford Appleton Laboratory, Chilton, Didcot, Oxon, U.K. ${ }^{o}$

E. Barberis, T. Dubbs, C. Heusch, M. Van Hook, B. Hubbard, W. Lockman, J.T. Rahn,

H.F.-W. Sadrozinski, A. Seiden

University of California, Santa Cruz, CA, USA ${ }^{p}$

J. Biltzinger, R.J. Seifert, O. Schwarzer, A.H. Walenta, G. Zech

Fachbereich Physik der Universität-Gesamthochschule Siegen, Federal Republic of Germany ${ }^{c}$

H. Abramowicz, G. Briskin, S. Dagan ${ }^{30}$, A. Levy ${ }^{31}$

School of Physics, Tel-Aviv University, Tel Aviv, Israel ${ }^{e}$

T. Hasegawa, M. Hazumi, T. Ishii, M. Kuze, S. Mine, Y. Nagasawa, M. Nakao, I. Suzuki, K. Tokushuku, S. Yamada, Y. Yamazaki

Institute for Nuclear Study, University of Tokyo, Tokyo, Japan ${ }^{g}$

M. Chiba, R. Hamatsu, T. Hirose, K. Homma, S. Kitamura, Y. Nakamitsu, K. Yamauchi

Tokyo Metropolitan University, Dept. of Physics, Tokyo, Japan ${ }^{g}$

R. Cirio, M. Costa, M.I. Ferrero, L. Lamberti, S. Maselli, C. Peroni, R. Sacchi, A. Solano, A. Staiano

Universita di Torino, Dipartimento di Fisica Sperimentale and INFN, Torino, Italy ${ }^{f}$

M. Dardo

II Faculty of Sciences, Torino University and INFN - Alessandria, Italy ${ }^{f}$

D.C. Bailey, D. Bandyopadhyay, F. Benard, M. Brkic, M.B. Crombie, D.M. Gingrich ${ }^{32}$, G.F. Hartner, K.K. Joo, G.M. Levman, J.F. Martin, R.S. Orr, C.R. Sampson, R.J. Teuscher

University of Toronto, Dept. of Physics, Toronto, Ont., Canada ${ }^{a}$

C.D. Catterall, T.W. Jones, P.B. Kaziewicz, J.B. Lane, R.L. Saunders, J. Shulman

University College London, Physics and Astronomy Dept., London, U.K. ${ }^{\circ}$

K. Blankenship, B. Lu, L.W. Mo

Virginia Polytechnic Inst. and State University, Physics Dept., Blacksburg, VA, USA ${ }^{q}$

W. Bogusz, K. Charchuła, J. Ciborowski, J. Gajewski, G. Grzelak, M. Kasprzak, M. Krzyżanowski,

K. Muchorowski, R.J. Nowak, J.M. Pawlak, T. Tymieniecka, A.K. Wróblewski, J.A. Zakrzewski, A.F. Żarnecki

Warsaw University, Institute of Experimental Physics, Warsaw, Poland ${ }^{j}$

M. Adamus

Institute for Nuclear Studies, Warsaw, Poland ${ }^{j}$

Y. Eisenberg ${ }^{30}$, U. Karshon ${ }^{30}$, D. Revel ${ }^{30}$, D. Zer-Zion

Weizmann Institute, Nuclear Physics Dept., Rehovot, Israel ${ }^{d}$

I. Ali, W.F. Badgett, B. Behrens, S. Dasu, C. Fordham, C. Foudas, A. Goussiou, R.J. Loveless, D.D. Reeder, S. Silverstein, W.H. Smith, A. Vaiciulis, M. Wodarczyk

University of Wisconsin, Dept. of Physics, Madison, WI, USA ${ }^{p}$

T. Tsurugai

Meiji Gakuin University, Faculty of General Education, Yokohama, Japan

S. Bhadra, M.L. Cardy, C.-P. Fagerstroem, W.R. Frisken, K.M. Furutani, M. Khakzad, W.B. Schmidke

York University, Dept. of Physics, North York, Ont., Canada ${ }^{a}$ 
${ }^{1}$ supported by Worldlab, Lausanne, Switzerland

2 also at IROE Florence, Italy

${ }^{3}$ now at Univ. of Salerno and INFN Napoli, Italy

${ }^{4}$ supported by EU HCM contract ERB-CHRX-CT93-0376

${ }^{5}$ now at Möbelhaus Kramm, Essen

${ }^{6}$ now a self-employed consultant

7 on leave of absence

${ }^{8}$ now at Institut für Hochenergiephysik, Univ. Heidelberg

${ }^{9}$ now also at University of Torino

10 Alfred P. Sloan Foundation Fellow

11 presently at Columbia Univ., supported by DAAD/HSPII-AUFE

12 now at Inst. of Computer Science, Jagellonian Univ., Cracow

13 now at Comma-Soft, Bonn

14 now at Univ. of Mainz

15 supported by DAAD and European Community Program PRAXIS XXI

${ }^{16}$ now at Dr. Seidel Informationssysteme, Frankfurt/M.

${ }^{17}$ supported by the European Community

18 now with OPAL Collaboration, Faculty of Physics at Univ. of Freiburg

19 now at SAS-Institut GmbH, Heidelberg

${ }^{20}$ also supported by DESY

21 now at GSI Darmstadt

22 also supported by NSERC

${ }^{23}$ now at Institute for Cosmic Ray Research, University of Tokyo

24 partially supported by CAM

25 now at Carleton University, Ottawa, Canada

${ }^{26}$ now at Department of Energy, Washington

27 now at HEP Div., Argonne National Lab., Argonne, IL, USA

${ }^{28}$ now at Oxford Magnet Technology, Eynsham, Oxon

${ }^{29}$ on leave and partially supported by DESY 1993-95

30 supported by a MINERVA Fellowship

31 partially supported by DESY

32 now at Centre for Subatomic Research, Univ.of Alberta, Canada and TRIUMF, Vancouver, Canada

a supported by the Natural Sciences and Engineering Research Council of Canada (NSERC)

$b \quad$ supported by the FCAR of Québec, Canada

c supported by the German Federal Ministry for Research and Technology (BMFT)

d supported by the MINERVA Gesellschaft für Forschung GmbH, and by the Israel Academy of Science

$e \quad$ supported by the German Israeli Foundation, and by the Israel Academy of Science

$f \quad$ supported by the Italian National Institute for Nuclear Physics (INFN)

$g \quad$ supported by the Japanese Ministry of Education, Science and Culture (the Monbusho) and its grants for Scientific Research

$h \quad$ supported by the Korean Ministry of Education and Korea Science and Engineering Foundation

i supported by the Netherlands Foundation for Research on Matter (FOM)

$j \quad$ supported by the Polish State Committee for Scientific Research (grant No. SPB/P3/202/93) and the Foundation for Polish- German Collaboration (proj. No. 506/92)

$k \quad$ supported by the Polish State Committee for Scientific Research (grant No. PB 861/2/91 and No. 22372 9102, grant No. PB 223769102 and No. PB 20092 9101)

$l \quad$ partially supported by the German Federal Ministry for Research and Technology (BMFT)

$m$ supported by the German Federal Ministry for Research and Technology (BMFT), the Volkswagen Foundation, and the Deutsche Forschungsgemeinschaft

$n \quad$ supported by the Spanish Ministry of Education and Science through funds provided by CICYT

o supported by the Particle Physics and Astronomy Research Council

$p \quad$ supported by the US Department of Energy

$q \quad$ supported by the US National Science Foundation 


\section{Introduction}

The investigation of strange particle production in neutral current, deep inelastic scattering (DIS) interactions could provide information about the $s$-quarks in the nucleon, about the boson-gluon fusion process and, above all, the parton fragmentation process. Strange particle production has been measured previously by experiments where the $\gamma^{*} p$ centre-of-mass energy, $W$, is at least one order of magnitude lower than at HERA [1, 2, 3, 团. The ratio of strange particle to light non-strange particle production of approximately 1:5 is ascribed to a reduced probability of strange quark creation in the parton fragmentation chain. In simulation programs based on the Lund scheme it is parametrised by the strange quark suppression factor $P_{s} / P_{u}$. Here $P_{s}$ and $P_{u}$ are the probabilities for creating $s-$ or $u, d$-quarks from the vacuum during the fragmentation process. A detailed review of our knowledge on heavy quark suppression is given in [5]. In hadron-hadron collisions an increasing $P_{s} / P_{u}$ is found with increasing centre-of-mass energy. Also indications of a dependence of the strangeness suppression factor on the region of phase space under investigation are reported. The values found vary between about 0.15 and 0.55 with a mean value close to 0.3 (see for example [6, 7]). The parameters for the hadronisation process in the present day electron-proton Monte Carlo event generators are obtained from fits to $e^{+} e^{-}$data and are assumed to be the same in DIS experiments due to jet universality. The longitudinal phase space of the $\gamma^{*} p$ interactions at HERA can be divided into three regions where different processes are expected to dominate. These processes also appear in $e^{+} e^{-}$or hadron-hadron scattering: a) the fragmentation region of the struck quark, which resembles that of one of the pairproduced quarks in $e^{+} e^{-}$annihilation experiments; b) the fragmentation of the proton remnant, which resembles the fragmentation in hadron colliders; and c) the hadronic centre-of-mass central rapidity region, where the colour flow between the struck quark and the proton remnant evolves. The latter region exists in both $e^{+} e^{-}$and hadron collider experiments. The acceptance of our central tracking detector allows us to study $K^{0}$ production in the fragmentation region of the struck quark and the central rapidity region. The part of the event which is well inside our detector acceptance is dominated by particles originating from the central rapidity region.

In about $10 \%$ of the DIS events no proton remnant is detected in the ZEUS detector, resulting in a large rapidity gap (LRG) between the acceptance limit in the proton direction and the first visible particle in the detector [8, 9]. The properties of these events are consistent with the assumption that the exchanged photon is scattered off a colourless object emitted by the proton. This object is generically called a pomeron. There exist indications that the pomeron has a partonic substructure [10, 8] but the nature of its constituents is still under investigation. A natural assumption is that they are quarks and gluons or a combination of both. It is expected that the strange quark content of the pomeron could affect the strange particle multiplicity in the final state of these events.

The investigation of strange particle production allows us to connect results from $e^{+} e^{-}$experiments and from hadron collider experiments. This paper is a first step of such a program. We compare the $K^{0}$ and $\Lambda$ multiplicities $\rrbracket^{\circ}$ and their momentum and angular distributions in the new kinematic region of HERA with extrapolations from Monte Carlo models based on the results of lower energy experiments. The $Q^{2}$ evolution of the $K^{0}$ multiplicity is studied. The production of $K^{0}$ 's in events with a large rapidity gap is compared to that of events without a large rapidity gap.

All studies are performed in the HERA laboratory frame and are restricted to a kinematic range where the tracking acceptance is high and well understood.

\footnotetext{
${ }^{1}$ Throughout this paper, a reference to a particle includes a reference to its antiparticle.
} 


\section{Experimental setup}

\section{HERA machine conditions}

The data were collected at the electron-proton collider HERA using the ZEUS detector during the 1993 running period. HERA collided $26.7 \mathrm{GeV}$ electrons with $820 \mathrm{GeV}$ protons. 84 bunches were filled for each beam and in addition 10 electron and 6 proton bunches were left unpaired for background studies. The typical electron and proton currents were $10 \mathrm{~mA}$ leading to a typical instantaneous luminosity of $6 \cdot 10^{29} \mathrm{~cm}^{-2} \mathrm{~s}^{-1}$. An integrated luminosity of $0.55 \mathrm{pb}^{-1}$ of data was collected in 1993 .

\section{The ZEUS detector}

ZEUS is a multipurpose, magnetic detector which has been described elsewhere [11]. Here we give a brief description concentrating on those parts of the detector relevant for the present analysis.

Charged particles are tracked by the inner tracking detectors which operate in a magnetic field of 1.43 $\mathrm{T}$ provided by a thin superconducting solenoid surrounding the tracking detectors. Immediately after the beampipe there is a cylindrical drift chamber, the vertex detector (VXD), which consists of 120 radial cells, each with 12 sense wires [12]. The achieved resolution is $50 \mu \mathrm{m}$ in the central region of a cell and $150 \mu \mathrm{m}$ near the edges. Surrounding the VXD is the central tracking detector (CTD) which consists of 72 cylindrical drift chamber layers, organised into 9 "superlayers" [13. Each superlayer consists either of wires parallel (axial) to the beam axis or of wires inclined at a small angle to give a stereo view. With the present understanding of the chamber, a spatial resolution of $260 \mu \mathrm{m}$ has been achieved. The hit efficiency of the chamber is greater than $95 \%$.

In events with charged particle tracks, using the combined data from both chambers, reconstructed primary vertex position resolutions of $0.6 \mathrm{~cm}$ in the $Z$ direction and $0.1 \mathrm{~cm}$ in the $X Y$ plane are measured 2 . The resolution in transverse momentum for full length tracks is $\sigma\left(p_{\mathrm{T}}\right) / p_{\mathrm{T}}=\sqrt{\left(0.005 p_{\mathrm{T}}\right)^{2}+(0.016)^{2}}$ $\left(p_{\mathrm{T}}\right.$ in $\left.\mathrm{GeV}\right)$.

The solenoid is surrounded by a high resolution uranium-scintillator calorimeter divided into three parts, forward (FCAL), barrel (BCAL) and rear (RCAL). Holes of $20 \times 20 \mathrm{~cm}^{2}$ in the centre of FCAL and RCAL are required to accommodate the HERA beam pipe. Each of the calorimeter parts is subdivided into towers which in turn are segmented longitudinally into electromagnetic (EMC) and hadronic (HAC) sections. A section of a tower is called a cell and is read out by two photomultiplier tubes. A detailed description of the calorimeter is given in [14].

For measuring the luminosity via the Bethe-Heitler process $e p \rightarrow e^{\prime} p^{\prime} \gamma$, as well as for tagging very small $Q^{2}$ processes, two lead-scintillator calorimeters are used [15. Bremsstrahlung photons emerging from the electron-proton interaction region at angles $\theta_{\gamma}^{\prime} \leq 0.5 \mathrm{mrad}$ with respect to the electron beam axis hit the photon calorimeter at $107 \mathrm{~m}$ from the interaction point (IP). Electrons emitted from the IP at scattering angles less than or equal to $6 \mathrm{mrad}$ and with energies between $20 \%$ and $90 \%$ of the incident electron energy are deflected by beam magnets and hit the electron calorimeter placed $35 \mathrm{~m}$ from the IP.

\section{HERA kinematics}

The kinematics of deep inelastic scattering processes at HERA, $e^{-} p \rightarrow e^{-} h$, where $h$ is the hadronic final state, can be described by the Lorentz invariant variables $Q^{2}, x$ and $y$. Here $-Q^{2}$ is the square of the four-momentum transfer between the incoming electron and the scattered electron; $x$, in the naïve quark-parton model, is the fractional momentum of the struck quark in the proton, and $y$ is the relative energy transfer of the electron to the hadronic system. The variables are related by $Q^{2}=s x y$, where $s$ is the squared invariant mass of the $e p$ system. $Q^{2}, x$ and $y$ can be calculated

${ }^{2}$ The ZEUS coordinate system is defined as right-handed with the $Z$ axis pointing in the proton beam direction and the $X$ axis horizontal pointing towards the centre of HERA. The polar angle $\theta$ is defined with respect to the $Z$-direction. 
from the kinematic variables of the scattered electron, from the hadronic final state variables, or from a combination of both. The optimal reconstruction method depends on the event kinematics and the detector resolution.

In this paper we use the double angle method [16] to calculate the $Q^{2}$ and $x$ variables:

$$
\begin{aligned}
Q_{D A}^{2} & =4 E_{e}^{2} \cdot \frac{\sin \gamma_{h}\left(1+\cos \theta_{e}\right)}{\sin \gamma_{h}+\sin \theta_{e}-\sin \left(\gamma_{h}+\theta_{e}\right)}, \\
x_{D A} & =\frac{E_{e}}{E_{p}} \cdot \frac{\sin \gamma_{h}+\sin \theta_{e}+\sin \left(\gamma_{h}+\theta_{e}\right)}{\sin \gamma_{h}+\sin \theta_{e}-\sin \left(\gamma_{h}+\theta_{e}\right)} .
\end{aligned}
$$

Here $E_{e}$ and $E_{p}$ are the initial electron and proton energies; $\theta_{e}$ is the electron scattering angle with respect to the incident proton direction and $\gamma_{h}$ is the polar angle of a massless object balancing the momentum vector of the scattered electron to satisfy four-momentum conservation. In the naïve quark-parton model $\gamma_{h}$ is the scattering angle of the struck quark. It is determined from the hadronic energy flow in the calorimeter:

$$
\cos \gamma_{h}=\frac{\left(\sum p_{X}\right)_{h}^{2}+\left(\sum p_{Y}\right)_{h}^{2}-\left(\sum E-p_{Z}\right)_{h}^{2}}{\left(\sum p_{X}\right)_{h}^{2}+\left(\sum p_{Y}\right)_{h}^{2}+\left(\sum E-p_{Z}\right)_{h}^{2}} .
$$

Here the sums run over all calorimeter cells which are not assigned to the scattered electron and $\left(p_{X}, p_{Y}, p_{Z}\right)$ is the momentum vector assigned to each cell of energy $E$. The cell angles are calculated from the geometric centres of the cells and the vertex position of the event.

Using the hadronic energy flow of the final state, $y$ can be calculated according to the Jacquet-Blondel method [17]:

$$
y_{J B}=\frac{1}{2 E_{e}} \sum_{h}\left(E-p_{Z}\right)_{h} .
$$

For background rejection we also calculate $y$ using the electron information:

$$
y_{e}=1-\frac{E_{e}^{\prime}}{2 E_{e}}\left(1-\cos \theta_{e}\right)
$$

where $E_{e}^{\prime}$ is the energy of the scattered electron. The square of the centre-of-mass energy of the virtual photon-proton system, $\gamma^{*} p$, is calculated using:

$$
W_{D A}^{2}=m_{p}^{2}+Q_{D A}^{2}\left(\frac{1}{x_{D A}}-1\right)
$$

where $m_{p}$ is the proton mass. We use the described methods for calculating the kinematic variables and do not mention them explicitly below except when necessary.

\section{Event selection}

\subsection{Trigger conditions}

The trigger is organised in three levels [11]. For DIS events, the first level trigger (FLT) requires at least one of three conditions of energy sums in the EMC calorimeter: the BCAL EMC energy exceeds $3.4 \mathrm{GeV}$; the RCAL EMC energy (excluding the innermost towers surrounding the beam pipe) exceeds $2.0 \mathrm{GeV}$; or the RCAL EMC energy (including those towers) exceeds $3.75 \mathrm{GeV}$.

The second level trigger (SLT) rejects proton beam-gas events by using the times measured in the calorimeter cells. The DIS trigger rate of the SLT is about one-tenth of the FLT DIS trigger rate. The loss of DIS events at the SLT is negligible. 
The third level trigger (TLT) has the full event information available and uses physics-based filters. It applies tighter timing cuts to suppress beam-gas background further and also rejects beam halo muons and cosmic muons. The TLT selects DIS event candidates by calculating:

$$
\delta=\sum_{i} E_{i} \cdot\left(1-\cos \theta_{i}\right)>20 \mathrm{GeV}-2 E_{\gamma},
$$

where $E_{i}$ and $\theta_{i}$ are the energy and the polar angle of the energy deposits in the central calorimeter. $E_{\gamma}$ is the energy measured in the photon calorimeter of the luminosity monitor. The summation runs over all energy deposits in the calorimeter cells. For fully contained DIS events $\delta \approx 2 E_{e}=53.4 \mathrm{GeV}$. Photoproduction events have low values of $\delta$ compared to DIS events because the scattered electron escapes in the beam pipe hole of the rear calorimeter. For events with $Q^{2}$ less than $\sim 4 \mathrm{GeV}^{2}$ the calorimeter cannot detect the scattered electron.

For events with the scattered electron detected in the calorimeter, the trigger acceptance was essentially independent of the DIS hadronic final state. It was greater than $97 \%$ for $Q^{2}>10 \mathrm{GeV}^{2}$ and independent of $Q^{2}$. A total of $7 \cdot 10^{6}$ events passed the TLT and was written to tape during the 1993 running period.

\subsection{Offline event selection}

The offline selection of DIS events is similar to that described in our earlier publication [18]. The characteristic signature of a DIS event is the scattered electron detected in the uranium scintillator calorimeter. The pattern of energy deposition in the calorimeter cells is used to identify an electron candidate. We use the following criteria to select a sample of DIS events:

- a scattered electron candidate has to be found with $E_{e}^{\prime}>5 \mathrm{GeV}$ and an impact point at the RCAL surface outside a square of $32 \times 32 \mathrm{~cm}^{2}$ centred on the beam line. This requirement ensures that the electromagnetic shower is fully contained within the calorimeter and its impact point can be reconstructed with sufficient accuracy;

- $y_{e}<0.95$ to reduce photoproduction background;

- $35 \mathrm{GeV}<\delta<60 \mathrm{GeV}$ to remove photoproduction events and to suppress events with hard initial state radiation;

- $-50 \mathrm{~cm}<Z<40 \mathrm{~cm}$, where $Z$ is the position of the event vertex reconstructed from the CTD. This requirement rejects beam-gas and cosmic ray events.

From Monte Carlo studies we find an average electron finding efficiency of $95 \%$ in the kinematic range considered, being above $98 \%$ in most of the kinematic range and dropping below $70 \%$ for high $y$ events. The purity is better than $96 \%$ for electron energies above $10 \mathrm{GeV}$ and drops to about $60 \%$ at high $y$. A total of 91000 events survive these criteria.

The particle multiplicity and the kinematics of particle production depend on $Q^{2}$ and $x$. We have restricted our analysis to a kinematic range in $Q^{2}, x$ and $y$ in which migration effects are small [18] and have little influence on the momentum and angular distributions of the $K^{0}$ 's and $\Lambda$ 's. We chose the following range:

- $10 \mathrm{GeV}^{2}<Q^{2}<640 \mathrm{GeV}^{2}$;

- $0.0003<x<0.01$;

- $y>0.04$.

The $Q^{2}$ and $x$ variables are calculated according to the double angle method and $y$ with the JacquetBlondel method. After applying these criteria to the previously selected sample we are left with 27500 events. 


\section{Monte Carlo simulation}

Monte Carlo event simulation is used to determine the acceptance and resolution of the ZEUS detector. The simulation is based on the GEANT 3.13 [19] program and incorporates the knowledge of the detector and the trigger.

\section{Simulation of normal DIS events}

Neutral current DIS events with $Q^{2}>4 \mathrm{GeV}^{2}$ were generated using the HERACLES 4.4 program [20] which incorporates first order electroweak corrections. The Monte Carlo program LEPTO 6.1 [21], interfaced to HERACLES via the program DJANGO 6.0 [22], was used to simulate QCD cascades and fragmentation. The parton cascade was modelled in different ways:

- the colour-dipole model including the boson-gluon fusion process (CDM) as implemented in the ARIADNE 4.03 [23] program was used. In this model coherence effects are implicitly included in the formalism of the parton cascade;

- matrix element calculations plus the parton shower option (MEPS) as implemented in LEPTO were used, where coherence effects in the final state cascade are included by angular ordering of successive parton emissions.

These models use the Lund string fragmentation [24] for the hadronisation phase as implemented in JETSET 7.3 [25].

For the CDM event sample the $\mathrm{MRSD}_{-}^{\prime}$ parton density parametrisation for the proton was used [26]. The GRV 27] parametrisation was used for the MEPS data set. These parametrisations describe reasonably the HERA measurements of the proton structure function $\mathrm{F}_{2}$ [28, 29].

The simulations predict that about $10 \%$ of the $K_{S}^{0}$ 's are produced in charm events and about $5 \%$ originate from sea quarks in the proton. The remaining $\sim 85 \%$ of the $K_{S}^{0}$ 's are created in the fragmentation chain depending on the actual value of the strange-quark suppression factor $P_{s} / P_{u}$. The parameters of the Monte Carlo models are set to their default values $\left(P_{s} / P_{u}=0.3\right)$. We have also generated events with $P_{s} / P_{u}=0.2$ as suggested in [6] and we have compared the predictions of the simulations with the measured rates. Since the MEPS model and the CDM model behave similarly when reducing the $P_{s} / P_{u}$ parameter, we only show the predictions with $P_{s} / P_{u}=0.2$ for the CDM model.

\section{Simulation of large rapidity gap DIS events}

Our previous study [8] shows that diffractive models, specifically POMPYT [30] and a model by Nikolaev and Zakharov [31] as implemented in our Monte Carlo program NZ [32], give adequate descriptions of the properties of the LRG events. We have used POMPYT and NZ event samples for our study of $K^{0}$ multiplicities in events with a large rapidity gap. The POMPYT Monte Carlo program uses an implementation of the Ingelman and Schlein model [33], describing high energy diffractive processes. In this model the virtual photon interacts with the constituents of the pomeron emitted by the proton. Factorisation is assumed in the sense that the pomeron emission and the pomeron structure are independent. The current version of POMPYT contains no strange quark constituents for the pomeron. The NZ Monte Carlo model on the other hand is non-factorisable. Here the virtual photon fluctuates into a $q \bar{q}$ or a $q \bar{q} g$ state and interacts with a colourless two-gluon system emitted by the proton. The $q \bar{q} g$ states were fragmented as if they were $q \bar{q}$ states and the flavours are generated in $90 \%$ of the cases as $(u, d)$ and in $10 \%$ as $s$. 


\section{$6 \quad$ Selection of $K_{S}^{0}$ and $\Lambda$ candidates}

$K_{S}^{0}$ particles are identified in the decay channel $K_{S}^{0} \rightarrow \pi^{+} \pi^{-}$and $\Lambda$ particles are detected in the channel $\Lambda \rightarrow p \pi^{-}$. Due to their lifetime of $\mathcal{O}\left(10^{-10} \mathrm{~s}\right)$ and their typical momenta of about $1 \mathrm{GeV}$ they have an average decay length of a few centimetres, which results in secondary vertices well separated from the primary event vertex.

Tracks are reconstructed using the CTD and the VXD. The track finding algorithm starts with hits in the outermost axial superlayers of the CTD. As the trajectory is followed inwards to the beam axis, more hits on the axial wires and from the VXD are assigned to the track. The resulting circle in the transverse plane is used for the pattern recognition in the stereo superlayers. The momentum is determined in a 5-parameter helix fit. Multiple Coulomb scattering in the beam pipe and in the outer walls of the VXD is taken into account in the evaluation of the covariance matrix.

The primary event vertex is determined from a $\chi^{2}$ fit performed with the tracks using the perigee parametrisation [34] and assuming that the tracks come from a common point in space. A track is considered not to be associated with the primary vertex if the $\chi^{2}$ for the primary vertex fit increases significantly when the track is included in the fit.

The systematic effects in the CTD are most serious for low $p_{\mathrm{T}}$ tracks and for tracks which traverse the inhomogeneous part of the magnetic field at the ends of the CTD. The reconstructed tracks used in this analysis were required to have a transverse momentum $p_{\mathrm{T}}>0.2 \mathrm{GeV}$ and a polar angle between $25^{\circ}<\theta<155^{\circ}$. In terms of pseudorapidity, $\eta=-\log (\tan (\theta / 2))$, this corresponds to $|\eta|<1.5$. This is the region where the CTD response and systematics are well understood.

\section{1 $K_{S}^{0}$ Identification}

To search for $K_{S}^{0}$, we examine pairs of oppositely charged tracks to find a secondary vertex. We refer to these tracks as daughter tracks. At least one of the daughter tracks is not allowed to be associated with the primary vertex and track pairs which do not intersect when projected into the transverse plane are rejected.

For each remaining track pair, we obtain the momentum of the $K_{S}^{0}$ candidate by calculating the momenta of the individual tracks at their intersection point and adding them. $K_{S}^{0}$ candidates with transverse momenta below $0.5 \mathrm{GeV}$ or above $4 \mathrm{GeV}$ or with directions of flight too near to the beam pipe, $|\eta|>1.3$, are removed.

The background in the mass region of the $K_{S}^{0}$ is reduced by applying the following criteria:

- $\cos \left(\alpha_{X Y}\right)>0.99$, where $\alpha_{X Y}$ is the angle in the transverse plane between the direction of flight of the $K_{S}^{0}$ candidate and its reconstructed momentum direction;

- the separation in $\mathrm{Z}$ between the two tracks at their $X Y$ intersection point has to be $|\Delta Z|<$ $2.5 \mathrm{~cm}$. The coordinates of the $K_{S}^{0}$ decay vertex are set to the $X Y$ coordinates of the intersection point of the track circles and the $Z$ coordinate is chosen to be in the centre between the closest approaches in $Z$ of the two track circles;

- the proper lifetime of the candidates, $c \tau=(L M c) / p$, has to be less than $10 \mathrm{~cm}$. Here $L$ is the decay length, $p$ is the momentum and $M$ is the invariant mass of the candidate;

- to reduce background arising from photon conversions into $e^{+} e^{-}$pairs, pairs of tracks considered as electrons must have an effective mass $M_{e e}>50 \mathrm{MeV}$ (see Fig. 1);

- to eliminate $\Lambda$ contamination of the $K_{S}^{0}$ signal, candidates with a mass hypothesis $M_{p \pi}<$ $1.12 \mathrm{GeV}$ are rejected (see Fig. 1).

Using these criteria (summarised in Tab. 1) we obtain the $K_{S}^{0}$ signal shown in Fig. 2a. We fit the $\pi^{+} \pi^{-}$mass spectrum with a Gaussian and a linear background in the region 0.4 to $0.6 \mathrm{GeV}$. The 
fitted mass is $497.4 \pm 0.3 \mathrm{MeV}$ and the standard deviation is $7.8 \pm 0.3 \mathrm{MeV}$. The mass value and width of the signal are well reproduced by the Monte Carlo simulations. In the signal region we find a total of $971 K_{S}^{0}$ mesons on top of a background of about $150 \pi \pi$-combinations. The $K_{S}^{0}$ signal region extends from 474 to $521 \mathrm{MeV}$. The average lifetime of the $K_{S}^{0}$ mesons was determined by fitting the exponential form $\exp \left(-c \tau / c \tau K_{S}^{0}\right)$ to the acceptance corrected $c \tau$ lifetime distribution. Here the $c \tau$ upper limit was relaxed to $20 \mathrm{~cm}$ and all other selection criteria were set to their default value. The result $c \tau_{K_{S}^{0}}^{0}=2.66 \pm 0.11 \pm 0.06 \mathrm{~cm}$ is consistent with the world average of $2.676 \pm 0.006 \mathrm{~cm}$ given in [35]. The systematic uncertainty includes the variation of the number of bins used in the fit and tightening or loosening the selection criteria.

\section{2 $\Lambda$ Identification}

The $\Lambda$ identification closely resembles the $K_{S}^{0}$ identification. The daughter track with the higher momentum is considered to be the proton. No daughter track is allowed to be associated with the primary vertex. The $c \tau$ upper limit is increased to $40 \mathrm{~cm}$ in order to account for the longer lifetime of the $\Lambda$. Requiring $M_{\pi \pi}<0.481 \mathrm{GeV}$ removes the background from $K_{S}^{0}$ mesons (see Fig. 1). Since there is no clear $\Lambda$ signal seen for candidates with $p_{\mathrm{T}}$ above $3.5 \mathrm{GeV}$, this value is chosen as the upper limit of the investigated momentum range.

Figure 2b shows the $\Lambda$ signal obtained. We fit the $p \pi$ mass spectrum from 1085 to $1185 \mathrm{MeV}$. The fit yields a mass of $1116.2 \pm 0.4 \mathrm{MeV}$ with a standard deviation of $3.0 \pm 0.5 \mathrm{MeV}$. The Monte Carlo simulation reproduces well the $\Lambda$ mass position and width. Within the signal region we find $80 \Lambda$ baryons and 18 background combinations. The signal region runs from 1107 to $1125 \mathrm{MeV}$. Of the 80 $\Lambda$ baryons, $(60 \pm 5) \%$ are $\bar{\Lambda}$ and the remaining $(40 \pm 5) \%$ are $\Lambda$. The determination of the average lifetime of the $\Lambda$ from the lifetime distribution gives $c \tau_{\Lambda}=7.3 \pm 2.2 \pm 0.5 \mathrm{~cm}$, consistent with the value of $7.89 \mathrm{~cm}$ given in 35.

\begin{tabular}{|l|c|c|}
\hline Selection parameters for candidates & $K_{S}^{0}$ & $\Lambda$ \\
\hline $\cos \left(\alpha_{X Y}\right)$ & $>0.99$ & $>0.99$ \\
$|\triangle Z|[\mathrm{cm}]$ & $<2.5$ & $<2.5$ \\
$\mathrm{c} \tau[\mathrm{cm}]$ & $<10$ & $<40$ \\
$M_{p \pi}[\mathrm{GeV}]$ & $>1.12$ & - \\
$M_{\pi \pi}[\mathrm{GeV}]$ & - & $<0.481$ \\
$M_{e e}[\mathrm{GeV}]$ & $>0.05$ & $>0.05$ \\
$p_{\mathrm{T} \text { daughter-tracks }}[\mathrm{GeV}]$ & $>0.2$ & $>0.2$ \\
$\theta_{\text {daughter-tracks }}\left[{ }^{\circ}\right]$ & {$[25,155]$} & {$[25,155]$} \\
No. of tracks from primary vertex & $\leq 1$ & 0 \\
\hline$\eta$ range & {$[-1.3,1.3]$} & {$[-1.3,1.3]$} \\
$p_{\mathrm{T}}[\mathrm{GeV}]$ range & {$[0.5,4.0]$} & {$[0.5,3.5]$} \\
\hline
\end{tabular}

Table 1: Selection criteria for $K_{S}^{0}$ and $\Lambda$ identification.

\section{Data correction}

This analysis uses two types of selection criteria. The first kind is event based and selects a reasonably pure sample of DIS events with minimal contamination from background (photoproduction, beam-gas, cosmic-ray events). The second kind of selection criteria is particle based and selects a sample of $K_{S}^{0}$ and $\Lambda$ particles from the event sample defined above. 
We find a $90 \%$ event selection efficiency, where we define the efficiency as the ratio of the number of Monte Carlo events passing all the event selection criteria (including those that restrict the kinematic range in $Q^{2}, x$ and $y$ ) to the total number of generated events in the restricted kinematic region.

We have restricted the $K^{0}$ and $\Lambda$ kinematic ranges to regions where our systematic uncertainties are small: their pseudorapidity is limited to $-1.3<\eta<1.3$ and their transverse momentum is restricted to a $p_{\mathrm{T}}$ between $0.5 \mathrm{GeV}$ and $4.0 \mathrm{GeV}(3.5 \mathrm{GeV})$ for $K^{0}$ 's $(\Lambda$ 's). We do not extrapolate our results to the full $p_{\mathrm{T}}$ and $\eta$ range in order not to be dominated by model predictions. The models are known to have uncertainties especially in the low $p_{\mathrm{T}}$ region and are not yet compared to particle properties in the proton fragmentation region of HERA events.

The $K_{S}^{0}$ and $\Lambda$ reconstruction efficiencies were determined as a function of $p_{\mathrm{T}}$ and $\eta$. For each particle type, the efficiency in a given $\left(p_{\mathrm{T}}, \eta\right)$ bin was defined as the ratio of the number of reconstructed particles in the bin to the number of generated particles in the bin. The $\eta$ and $p_{\mathrm{T}}$ resolutions are less than $5 \%$ of the bin width chosen for the plots and show no systematic shifts. The DIS Monte Carlo events that passed all the selection criteria were used for these calculations. The $K_{S}^{0}$ reconstruction efficiency in the kinematic region considered varies between $20 \%$ for low $p_{\mathrm{T}}$ and $55 \%$ for $p_{\mathrm{T}}$ above $1.5 \mathrm{GeV}$. The efficiency varies in $\eta$ from $30 \%$ around $\eta= \pm 1.3$ to $40 \%$ for $K_{S}^{0}$ 's moving transversely to the beam direction $(\eta=0)$. The $\Lambda$ reconstruction efficiency varies between $5 \%$ for low transverse momentum and approaches $20 \%$ for high $p_{\mathrm{T}}$. The efficiency varies in $\eta$ between $10 \%$ and $15 \%$. The largest loss of true $K_{S}^{0}$ 's and $\Lambda$ 's results from the collinearity requirement $\left(\alpha_{X Y}\right)$ and the requirement that daughter tracks are unassociated with the event vertex. Each requirement rejects about $25 \%$ of the candidates if no other selection criterion is applied.

The $K^{0}(\Lambda)$ measurements are corrected for the above efficiencies as well as for the branching ratios $K^{0}$ to $K_{S}^{0}$ and $K_{S}^{0} \rightarrow \pi^{+} \pi^{-} \quad(\Lambda \rightarrow p \pi)$ [35]. No corrections were made to the measurements for migrations and initial state radiation effects since the changes predicted from Monte Carlo studies are small. Instead we include these effects in our systematic error analysis (see section 9).

The analysis procedure was checked using the reconstructed CDM (MEPS) Monte Carlo events as if they were data events and correcting them with the efficiencies obtained with the MEPS (CDM) samples. The corrected Monte Carlo distributions agreed at the $5 \%$ level with the generated distributions. For the comparison of $K^{0}$ production in events with and without a large rapidity gap, the twodimensional $\left(p_{\mathrm{T}}, \eta\right)$ efficiencies were determined from the standard DIS Monte Carlo events satisfying the additional requirement $W>140 \mathrm{GeV}$ (see section 8.2 for details). This corresponds to a restriction to $y>0.22$. Both non-LRG (NRG) and LRG data samples were corrected with the same efficiencies. It has been checked that the corrected and generated $K_{S}^{0}$ distributions of the LRG Monte Carlo events agree well when using the efficiencies of those Monte Carlo sets.

The ratio of $K^{0}$ to charged particle multiplicity, $\mathrm{N}\left(K^{0}\right) / \mathrm{N}($ tracks), is investigated below. The charged particle multiplicity, $\mathrm{N}$ (tracks), is determined for charged particles originating at the primary vertex and produced in the restricted kinematic range $|\eta|<1.3$ and $p_{\mathrm{T}}>0.2 \mathrm{GeV}$. The number of reconstructed tracks is corrected for tracking inefficiencies, wrong assignments by the vertex finding routine to the decay products of long lived particles and pair conversions by using standard Monte Carlo techniques. The Monte Carlo corrections for particle based selection criteria were below $10 \%$.

\section{Results}

\section{1 $K^{0}$ and $\Lambda$ multiplicity distributions}

Figure 3 shows the differential $K^{0}$ multiplicity as a function of $p_{\mathrm{T}}$ and $\eta$. The inner error bars are statistical errors and the outer ones statistical and systematic errors added in quadrature. The distributions are normalised by the number of events $N_{e v}$. The predictions of the CDM and the MEPS models are overlaid. The two curves for the CDM sample are generated with different strange-quark suppression factors $P_{s} / P_{u}$. The predicted multiplicity for the default strange-quark suppression factor 
of 0.3 is higher than measured. Using the smaller suppression factor of 0.2 reduces the predicted multiplicity to a value closer to that observed in the data. Both parameters give a reasonable description of the measured shapes.

For events with $10<Q^{2}<640 \mathrm{GeV}^{2}, 0.0003<x<0.01$ and $y>0.04$, the number of neutral kaons per event with $0.5<p_{\mathrm{T}}<4.0 \mathrm{GeV}$ and $|\eta|<1.3$ is $0.289 \pm 0.015 \pm 0.014$. The first error is statistical, the second error is systematic. A function of the form $C_{1} / p_{\mathrm{T}} \cdot \exp \left(C_{2} p_{\mathrm{T}}\right)$ fits well the measured $1 / N_{e v} \cdot d N\left(K^{0}\right) / d p_{T}^{2}$ distribution as a function of $p_{\mathrm{T}}$ over the $p_{\mathrm{T}}$ range shown in Fig. 3 . $C_{1}$ and $C_{2}$ are constants. The slope, $C_{2}$, of the $p_{\mathrm{T}}$ distribution for the $K^{0}$ 's is $-1.31 \pm 0.09 \pm 0.06$ $\mathrm{GeV}^{-1}$. These values, together with the predictions from Monte Carlo models, are listed in Tab. 2 . According to Monte Carlo studies, the fraction of $K^{0}$ 's produced in the restricted $p_{\mathrm{T}}$ and $\eta$ range is $23 \%$ of the total number of $K^{0}$ 's produced in the final state.

\begin{tabular}{|l|l|l|}
\hline & $\mathrm{N}\left(K^{0}\right) /$ event & $p_{\mathrm{T}}$ slope $\left[\mathrm{GeV}^{-1}\right]$ \\
\hline Data & $0.289 \pm 0.015 \pm 0.014$ & $-1.31 \pm 0.09 \pm 0.06$ \\
CDM & & \\
$\quad$ with $P_{s} / P_{u}=0.3$ & $0.342 \pm 0.005$ & $-1.40 \pm 0.05$ \\
$\quad$ with $P_{s} / P_{u}=0.2$ & $0.264 \pm 0.003$ & $-1.37 \pm 0.04$ \\
MEPS & & $-1.36 \pm 0.05$ \\
$\quad$ with $P_{s} / P_{u}=0.3$ & $0.348 \pm 0.006$ & \\
\hline
\end{tabular}

Table 2: Results of the $K^{0}$ measurement for events with $10<Q^{2}<640 \mathrm{GeV}^{2}, 0.0003<x<0.01$, $y>0.04$ and for a $K^{0}$ with $0.5<p_{\mathrm{T}}<4.0 \mathrm{GeV}$ and $|\eta|<1.3$. The two CDM samples have been generated with a different strange-quark suppression factor $P_{s} / P_{u}$.

Figures Ga, b show the differential $\Lambda$ multiplicity as a function of the transverse momentum and the pseudorapidity. The predictions of the CDM and the MEPS Monte Carlo are also displayed in Fig. 4 . The two CDM curves correspond to samples generated with different strange-quark suppression factors $P_{s} / P_{u}$. The number of $\Lambda$ 's with $0.5<p_{\mathrm{T}}<3.5 \mathrm{GeV}$ and $|\eta|<1.3$ produced per event is $0.038 \pm 0.006 \pm 0.002$ for events with $10<Q^{2}<640 \mathrm{GeV}^{2}, 0.0003<x<0.01, y>0.04$. The measured slope of the $p_{\mathrm{T}}$ distribution of the $\Lambda$ is $-1.4 \pm 0.3 \pm 0.1 \mathrm{GeV}^{-1}$, which, due to the large statistical uncertainty, is still in agreement with the model predictions. These values, together with the predictions of the models are listed in Tab. 3. Monte Carlo studies predict that $16 \%(25 \%)$ of the total number of $\Lambda$ 's $(\bar{\Lambda}$ 's $)$ will be inside this restricted $p_{\mathrm{T}}$ and $\eta$ region.

The measured $K^{0}$ and $\Lambda$ multiplicities seem to be better described by a model with a strangeness suppression factor of 0.2 .

\begin{tabular}{|l|l|l|}
\hline & $\mathrm{N}(\Lambda) /$ event & $p_{\mathrm{T}}$ slope $\left[\mathrm{GeV}^{-1}\right]$ \\
\hline Data & $0.038 \pm 0.006 \pm 0.002$ & $-1.4 \pm 0.3 \pm 0.1$ \\
CDM & & \\
$\quad$ with $P_{s} / P_{u}=0.3$ & $0.066 \pm 0.003$ & $-1.04 \pm 0.07$ \\
with $P_{s} / P_{u}=0.2$ & $0.050 \pm 0.002$ & $-1.00 \pm 0.06$ \\
MEPS & & $-0.98 \pm 0.06$ \\
$\quad$ with $P_{s} / P_{u}=0.3$ & $0.068 \pm 0.003$ & \\
\hline
\end{tabular}

Table 3: Results of the $\Lambda$ measurement for events with $10<Q^{2}<640 \mathrm{GeV}^{2}, 0.0003<x<0.01$, $y>0.04$ and for a $\Lambda$ with $0.5<p_{\mathrm{T}}<3.5 \mathrm{GeV},|\eta|<1.3$. The two CDM samples have been generated with a different strange-quark suppression factor $P_{s} / P_{u}$. 
We have studied the mean $K^{0}$ multiplicity and the ratio of $K^{0}$ to charged particle multiplicities $\mathrm{N}\left(K^{0}\right) / \mathrm{N}$ (tracks) as a function of the $Q^{2}$ of the event. In order to stay in the region of uniform acceptance given by the inner tracking detector geometry and the analysis cuts, we restrict this study to events with $-1.5<\eta_{\gamma_{h}}<0$. Figure 5 shows the distribution of our event sample in the $\left(x, Q^{2}\right)$ plane. The lines of constant $\gamma_{h}$ delimiting the accepted events and the $Q^{2}$ bins chosen for this study are shown. In those bins the variables $Q^{2}$ and $W$ are correlated: as $Q^{2}$ increases from $10 \mathrm{GeV}^{2}$ to $200 \mathrm{GeV}^{2}$, the mean value of $W$ increases from $110 \mathrm{GeV}$ to $160 \mathrm{GeV}$. Figure 6a, b show the mean $K^{0}$ multiplicity and the ratio $\mathrm{N}\left(K^{0}\right) / \mathrm{N}$ (tracks) in the selected bins plotted versus the mean $Q^{2}$ of the bins. The number of charged particles does not include secondary particles from $K^{0}$ and $\Lambda$ decays and from weakly decaying particles with a lifetime $>10^{-8} \mathrm{~s}$. A slight increase of the $K^{0}$ multiplicity and a constant behaviour of $\mathrm{N}\left(K^{0}\right) / \mathrm{N}$ (tracks) are observed. We have included the predictions from the CDM and MEPS Monte Carlo samples, which describe the data reasonably well. A study at the Monte Carlo generator level shows that the mean $K^{0}$ multiplicity is independent of $Q^{2}$ for fixed $W$. Since data and Monte Carlo agree over a wide range of $Q^{2}$, we conclude that the mean $K^{0}$ multiplicity of our data also shows no $Q^{2}$ dependence at fixed $W$ within the accuracy of these data. Furthermore the ratio of $K^{0}$ to charged particle multiplicities is observed to be constant and thus within our experimental errors this ratio does not depend on the kinematic variables in the region under study. Therefore we attribute our observed increase of $K^{0}$ multiplicity with $Q^{2}$ to the increase of the corresponding $W$ values.

\section{2 $K^{0}$ production in events with a large rapidity gap}

The DIS data sample is a mixture of non-diffractive and diffractive events. We have searched for differences in $K^{0}$ production in these event types. Following our earlier publications [8, 36], we separate a non-rapidity gap event sample (NRG) and a LRG event sample using $\eta_{\max }$. $\eta_{\max }$ is the largest pseudorapidity of any calorimeter cluster in an event, where a cluster is defined as an isolated set of adjacent cells with summed energy above $400 \mathrm{MeV}$. The NRG sample is selected by $\eta_{\max }>1.5$. It is dominated by non-diffractive events. The requirement $\eta_{\max }<1.5$ selects a LRG sample which is dominated by diffractive events. The standard non-diffractive DIS models (CDM, MEPS) give a reasonable description of the $\eta_{\max }$ distribution for values above 1.5 but cannot account for the excess of events at lower values (see Fig. 7a). Values of $\eta_{\max }>4.3$, which are outside the calorimeter acceptance, occur when energy is deposited in many contiguous cells around the beam pipe in the proton direction. An admixture of about $10 \%-20 \%$ of diffractive events generated with the NZ or POMPYT Monte Carlo programs to the non-diffractive Monte Carlo sample gives a reasonable description of the $\eta_{\max }$ distribution. The background of non-diffractive DIS events in the LRG sample is estimated to be $7 \%$ [8]. Less than $10 \%$ of the NRG DIS event sample are diffractive events. Figure $7 \mathrm{~b}$ shows the $\eta_{\max }$ distribution for those events which have a $K_{S}^{0}$ candidate in the signal band. The $\eta_{\max }$ distribution of events from one of the non-diffractive (CDM) and from one of the diffractive (NZ) Monte Carlo samples is also shown. The excess of $K_{S}^{0}$ candidates over predictions from the CDM model for $\eta_{\max }$ $<1.5$ represents the $K_{S}^{0}$ production in diffractive events.

As discussed elsewhere [8, 36], the acceptances for diffractive events selected by the LRG requirement $\left(\eta_{\max }\right)$ and for NRG events are flat with respect to $W$ and $Q^{2}$ for $W>140 \mathrm{GeV}$. We have therefore restricted our comparison to events with $W>140 \mathrm{GeV}$. After this additional requirement, 11000 NRG events and $940 \mathrm{LRG}$ events remain. In the LRG sample we find in the signal region $18 K_{S}^{0}$ candidates over a background of 2 candidates.

Figure 8 shows the differential $K^{0}$ multiplicity as a function of the transverse momentum and pseudorapidity for NRG and for LRG DIS events separately. The results in this subsection are not corrected for either the $\eta_{\max }$ or the $W$ selection criteria. The predictions of the standard DIS Monte Carlo programs (CDM and MEPS) and the diffractive DIS Monte Carlo programs (POMPYT and NZ) are shown. The $p_{\mathrm{T}}$ distributions have similar shapes in both data subsamples, although the multiplicity is lower for the LRG DIS events. Within the limited statistics of the data, both diffractive models 
give a reasonable description of the $K^{0}$ multiplicities in LRG events.

Since the invariant mass of the measured hadronic system in LRG events is smaller than in NRG events, a reduced $K^{0}$ rate is expected in the diffractive events. We have compared the $K^{0}$ multiplicity with the charged particle multiplicity for both subsamples. Table 4 lists the $K^{0}$ multiplicity and the ratio of the $K^{0}$ to charged particle multiplicity for NRG and LRG DIS events and for the Monte Carlo samples. If one subtracts the diffractive background, which, as seen from Fig. 0, is still present in the NRG DIS sample, the quoted $K^{0}$ multiplicity in the non-diffractive DIS sample increases by $5 \%$. The ratios of $K^{0}$ 's to charged tracks for both data samples are consistent with each other. Thus, within the limited statistics, these results give no indication of any additional strange quark enhancement or suppression in the production mechanism of the LRG final state.

\begin{tabular}{|c|l|l|l|}
\hline & Data type & $\mathrm{N}\left(K^{0}\right) /$ event & $\mathrm{N}\left(K^{0}\right) / \mathrm{N}($ tracks $)$ \\
\hline $\begin{array}{c}\eta_{\max }>1.5 \\
\text { NRG }\end{array}$ & ZEUS data & $0.344 \pm 0.023 \pm 0.025$ & $0.077 \pm 0.006 \pm 0.008$ \\
& $\begin{array}{l}\text { CDM } \\
\text { with } P_{s} / P_{u}=0.3 \\
\quad \text { with } P_{s} / P_{u}=0.2\end{array}$ & $0.396 \pm 0.009$ & $0.095 \pm 0.003$ \\
& MEPS & & $0.071 \pm 0.003$ \\
& with $P_{s} / P_{u}=0.3$ & $0.375 \pm 0.009$ & $0.096 \pm 0.003$ \\
\hline$\eta_{\max }<1.5$ & ZEUS data & $0.156 \pm 0.047 \pm 0.007$ & $0.071 \pm 0.021 \pm 0.007$ \\
LRG & POMPYT & $0.106 \pm 0.010$ & $0.058 \pm 0.006$ \\
& NZ & $0.173 \pm 0.017$ & $0.073 \pm 0.007$ \\
\hline
\end{tabular}

Table 4: The $K^{0}$ multiplicity and the ratio of the $K^{0}$ and charged particle multiplicities for NRG and LRG DIS events. The predictions of five Monte Carlo samples are listed. The diffractive samples are generated with a strangeness suppression factor $P_{s} / P_{u}=0.3$.

\section{$9 \quad$ Study of systematic errors}

We have investigated several sources of systematic errors for our measurements of the $K^{0}$ and $\Lambda$ production rates.

1) The sensitivity of the results with respect to the track and primary vertex reconstruction methods was determined by repeating the analysis with a modified version of the reconstruction package. The differences seen are at the $5 \%$ level for the multiplicity distributions. No systematic effect is apparent. The ratio of $K^{0}$ to charged particle multiplicity is similarly unaffected.

2) The sensitivity of the results on the choice of the $K_{S}^{0}$ and $\Lambda$ selection criteria has been investigated by varying them by $\pm 25 \%$ of their nominal values. The uncertainty in the results from the DIS event selection was determined by repeating the analysis with different electron finding algorithms and by varying the event selection criteria by reasonable values. The systematic error from those sources is about $5 \%$ except for the highest $\eta$ and $p_{\mathrm{T}}$ points in the multiplicity distributions and for the results of the LRG event analysis, where the error is up to $15 \%$. The mean particle multiplicities per event show lower systematic errors $(3 \%)$ than the bin by bin errors in the figures.

3) Uncertainties from events rejected by the DIS event selection criteria and event migration effects were determined by detailed Monte Carlo studies of the $K^{0}$ and $\Lambda$ production in the events migrating into and out of the selected $Q^{2}, x, y$ range. The $K^{0}$ and $\Lambda$ rate of events migrating into this range is comparable to that of events migrating out. The uncertainty from these sources is at the $5 \%$ level. The additional kinematic restriction of $W>140 \mathrm{GeV}$ for the LRG comparison introduces a higher uncertainty $(7 \%)$ for the results. The mean particle multiplicities show a $2 \%$ uncertainty for NRG DIS events and $5 \%$ for the LRG DIS events. 
4) We determined a photoproduction contamination in the event sample of $2.5 \%$. The event sample which was kinematically restricted to $W>140 \mathrm{GeV}$ contains a higher background of $3.5 \%$ as shown in [18, 28]. We have estimated how these photoproduction events affect our analysis by studying the stability of the results when varying the scattered electron energy and the $\delta$ selection criterion. We quote an uncertainty from this source of $3 \%$. The influence on the results from initial state radiative events not removed by the $\delta$ requirement is below $3 \%$ except for the lowest $\eta$ point in Fig. $3 \mathrm{~b}$ where it is $15 \%$.

5) The $K^{0}$ multiplicity versus $Q^{2}$ is rather sensitive to the background below the $M_{\pi \pi}$ signal. The combinatorial background increases with $Q^{2}$ due to the observed higher particle multiplicity in events with higher $Q^{2}$. Also migration effects are non-negligible. Both effects together may induce variations to the measured values between $-11 \%$ and $+3 \%$ depending on the $Q^{2}$ bin and on the Monte Carlo simulations used to determine them. We include an overall systematic error of $10 \%$ to our results from these sources.

6) The results for the ratio of the $K^{0}$ multiplicity to the charged particle multiplicity are affected by uncertainties similar to those for the $K^{0}$ multiplicity alone. The variations resulting from different correction procedures of calculating the mean charged track multiplicity or from using different Monte Carlo samples for the correction are within a few percent. The relative changes of the ratios of $K^{0}$ to charged particle multiplicities for the NRG and the LRG data samples are below $5 \%$ when different $p_{\mathrm{T}}$ ranges for the charged particles are considered.

7) The strange quark density of the proton structure function does not affect our acceptance corrections.

\section{Summary and discussion}

We have measured the $K^{0}$ and $\Lambda$ multiplicities for deep inelastic ep scattering events at $\sqrt{s}=296 \mathrm{GeV}$ with $10 \mathrm{GeV}^{2}<Q^{2}<640 \mathrm{GeV}^{2}, \quad 0.0003<x<0.01$ and $y>0.04$ in the ZEUS experiment at HERA. We have restricted the analysis to the $K^{0}$ and $\Lambda$ kinematic region $p_{\mathrm{T}}>0.5 \mathrm{GeV}$ and $|\eta|<1.3$. About $23 \%(20 \%)$ of the $K^{0}(\Lambda)$ are predicted to be produced within this kinematic range.

In this kinematic range the mean number of $K^{0}(\Lambda)$ per event is $0.289 \pm 0.015 \pm 0.014(0.038 \pm 0.006$ $\pm 0.002)$. The results on particle production from lower energy $e^{+} e^{-}$data, which are incorporated in the current DIS Monte Carlo simulation programs (i.e., strange quark suppression factor $P_{s} / P_{u}=0.3$ ), predict higher $K^{0}$ and $\Lambda$ multiplicities than those observed in the data. Using a smaller value of 0.2 reduces the predicted multiplicity and gives a better agreement with the data, especially for $\Lambda$ production. Nevertheless, with $P_{s} / P_{u}=0.2$ the prediction for the $\Lambda$ multiplicity is still higher, while the prediction for the $K^{0}$ multiplicity is lower than the measured values. The Monte Carlo models allow an adjustment of the production rates of the different particle types by changing other parameters, like the ratio of diquarks to single quarks created from the sea, $P_{q q} / P_{q}$, as well as the suppression factor for strange diquarks, $\left(P_{u s} / P_{u d}\right) /\left(P_{s} / P_{d}\right)$. Our results indicate the need for tuning these parameters which requires a detailed measurement of the ratios of pions, kaons, lambdas and protons over a larger kinematic range. This is beyond the scope of this paper. The shapes of the distributions for $K^{0}$ 's and $\Lambda$ 's are described by both models and do not depend on the chosen parameter $P_{s} / P_{u}$.

The mean $K^{0}$ multiplicity of our data shows no indication for a $Q^{2}$ dependence at fixed $W$. Also, the ratio of $K^{0}$ to charged particles is observed to be independent of the kinematic variables in the range studied.

We observe $K^{0}$ production in DIS events with a large rapidity gap with respect to the proton direction. The $K^{0}$ multiplicity in LRG events is approximately a factor of two lower than in non-diffractive DIS events. The ratio of $K^{0}$ to charged particles is found to be the same in both samples. Thus we observe no additional enhancement or suppression of neutral kaon production in events with a large rapidity gap compared to events without a gap. 


\section{Acknowledgements}

The strong support and encouragement by the DESY Directorate have been invaluable. The experiment was made possible by the inventiveness and diligent efforts of the HERA machine group who continued to run HERA most efficiently during 1993.

The design, construction and installation of the ZEUS detector have been made by the ingenuity and dedicated efforts of many people from the home institutes who are not listed here. Their contributions are acknowledged with great appreciation. We also gratefully acknowledge the support of the DESY computing and network services.

\section{References}

[1] N.J. Baker et al., Phys. Rev. D34 (1986) 1251.

[2] V.V. Ammosov et al., Phys. Lett. B93 (1980) 210.

[3] S. Willocq et al., Z. Phys. C53 (1992) 207.

[4] G.T. Jones et al., Z. Phys. C57 (1993) 197.

[5] A.K. Wróblewski, Proceedings of the 25th International Conference on HEP, Singapore 1990, 125 .

[6] E665 Collaboration, M.R. Adams et al., Z. Phys. C61 (1994) 539.

[7] G.T. Jones et al., Z. Phys. C27 (1985) 43;

F. Abe et al., Phys. Rev. D40 (1989) 3791;

M. Arneodo et al., Z. Phys. C34 (1987) 283;

A. Breakstone et al., Phys. Lett. B135 (1984) 510;

CELLO Collaboration, H.J. Behrend et al., Z. Phys. C46 (1990) 397;

DELPHI Collaboration, P. Abreu et al, CERN-PPE-94-130 (1994);

HRS Collaboration, M. Derrick et al., Phys. Rev. D35 (1987) 2639;

HRS Collaboration, S. Abachi et al., Phys. Rev. D41 (1990) 2045;

JADE Collaboration, W. Bartel et al., Z. Phys. C20 (1983) 187;

OPAL Collaboration, G. Alexander et al., Phys. Lett. B264 (1991) 467;

TASSO Collaboration, M. Althoff et al., Z. Phys. C27 (1985) 27;

TASSO Collaboration, W. Braunschweig et al., Z. Phys. C47 (1990) 167.

[8] ZEUS Collaboration, M. Derrick et al., Phys. Lett. B332 (1994) 228.

[9] H1 Collaboration, T. Ahmed et al., Nucl. Phys. B429 (1994) 477.

[10] UA8 Collaboration, A. Brandt et al., Phys. Lett. B297 (1992) 417.

[11] ZEUS Collaboration, The ZEUS Detector, Status Report 1993, DESY 1993.

[12] C. Alvisi et al., Nucl. Instr. and Meth. A305 (1991) 30.

[13] N. Harnew et al., Nucl. Instr. and Meth. A279 (1989) 290;

B. Foster et al., Nucl. Phys. B (Proc. Suppl.) 32 (1993) 181;

B. Foster et al., Nucl. Instr. and Meth. A338 (1994) 254.

[14] A. Andresen et al., Nucl. Instr. and Meth. A309 (1991) 101;

A. Bernstein et al., Nucl. Instr. and Meth. A336 (1993) 23;

A. Caldwell et al., Nucl. Instr. and Meth. A321 (1992) 356. 
[15] J. Andruszków et al., DESY 92-066.

[16] S. Bentvelsen, J. Engelen and P. Kooijman, HERA Workshop 1991, Vol. 1, p. 23.

[17] F. Jacquet and A. Blondel, 1979 Proc. Study of an ep Facility in Europe, ed. U. Amaldi, 79, 48 p. 391.

[18] ZEUS Collaboration, M. Derrick et al., Phys. Lett. B316 (1993) 412.

[19] R. Brun et al., GEANT3, CERN DD/EE/84-1 (1987).

[20] A. Kwiatkowski, H. Spiesberger and H.-J. Möhring, Proc. of Workshop at HERA Vol. 3 DESY, (1982) 1294.

[21] G. Ingelman, Proceedings of the 1991 Workshop on Physics at HERA, DESY Vol. 3 (1992) 1366.

[22] K. Charchuła, G. Schuler and H. Spiesberger, CERN-TH. 7133/94.

[23] L. Lönnblad, Comput. Phys. Comm. 71 (1992) 15.

[24] B. Andersson et al., Phys. Rep. 97 (1983) 31.

[25] T. Sjöstrand, Comput. Phys. Comm. 39 (1986) 347.

[26] A.D. Martin, W.J. Stirling and R.G. Roberts, Phys. Lett. B306 (1993) 145.

[27] M. Glück, E. Reya and A. Vogt, Phys. Lett. B306 (1993) 391.

[28] ZEUS Collaboration, M. Derrick et al., Z. Phys. C65 (1995) 379.

[29] H1 Collaboration, I. Abt et al., Nucl. Phys. B407 (1993) 515.

[30] P. Bruni and G. Ingelman, Proceedings of the Europhysics Conference on HEP, Marseille, France, (1993) 595.

[31] N.N. Nikolaev and B.G. Zakharov, Z. Phys. C53 (1992) 331.

[32] A. Solano, Ph.D. Thesis, University of Torino 1993, unpublished;

A. Solano, Proceedings of the International Conference on Elastic and Diffractive Scattering, Nucl. Phys. B (Proc. Suppl.) 25 (1992) 274.

[33] G. Ingelman and P. Schlein, Phys. Lett. B152 (1985) 256.

[34] P. Billoir and S. Qian, Nucl. Instr. and Meth. A311 (1992) 139;

S. Qian, Nucl. Instr. and Meth. A350 (1994) 618.

[35] Particle Data Group, L. Montanet et al., Phys. Rev. D50 (1994) 1173.

[36] ZEUS Collaboration, M. Derrick et al., Phys. Lett. B315 (1993) 481. 


\section{ZEUS 1993}

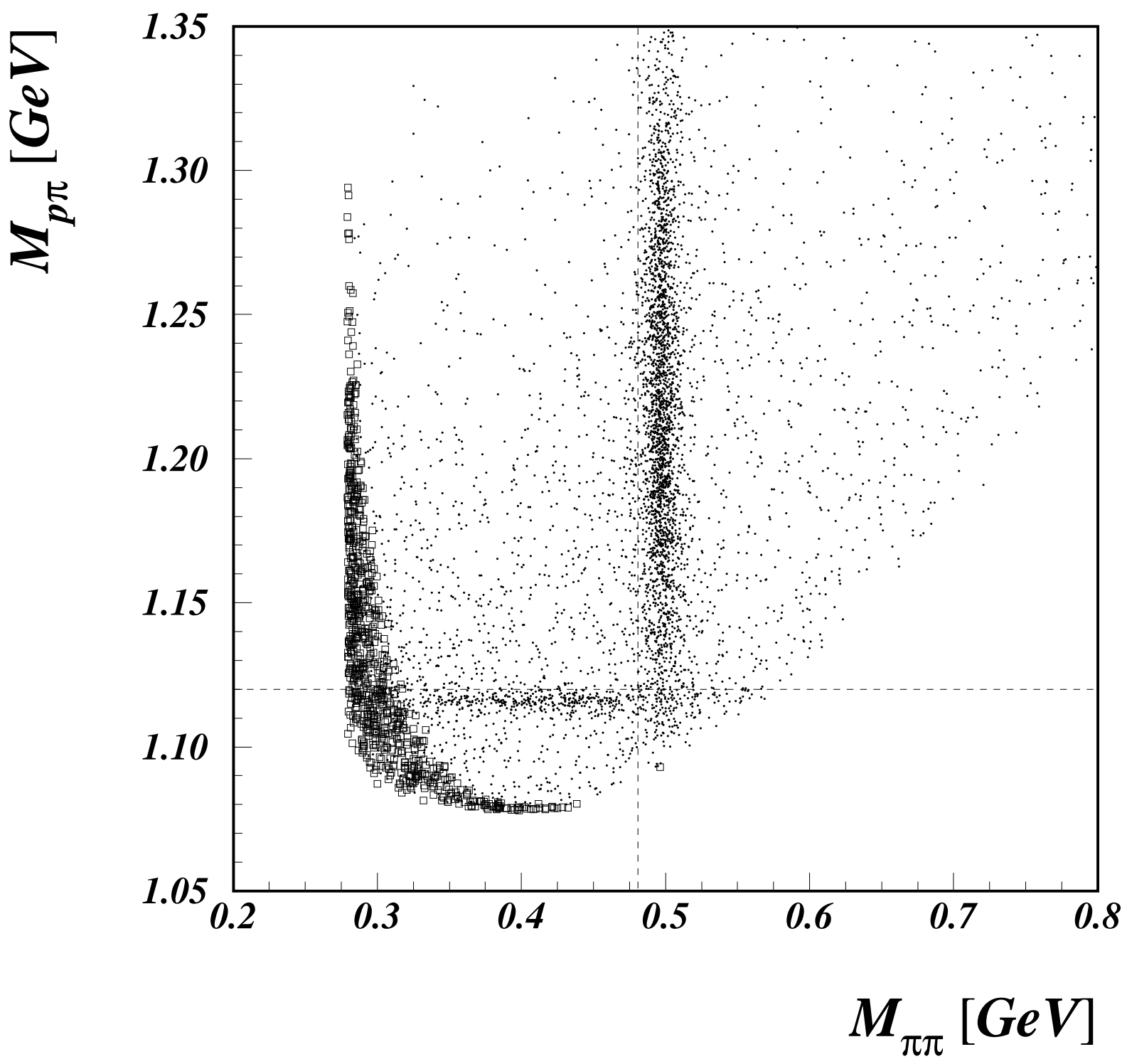

Figure 1: Invariant masses for two particle combinations assuming a $p \pi$ versus a $\pi \pi$ mass hypothesis. The background to the $K_{S}^{0}$ signal is reduced by removing the candidates with $M_{p \pi}<1.12 \mathrm{GeV}$. The background to the $\Lambda$ signal is reduced by removing candidates with $M_{\pi \pi}>0.481 \mathrm{GeV}$. The dashed lines correspond to these mass values. Candidates for photon conversions removed by requiring $M_{e e}<50 \mathrm{MeV}$ are indicated by squares. 
ZEUS 1993
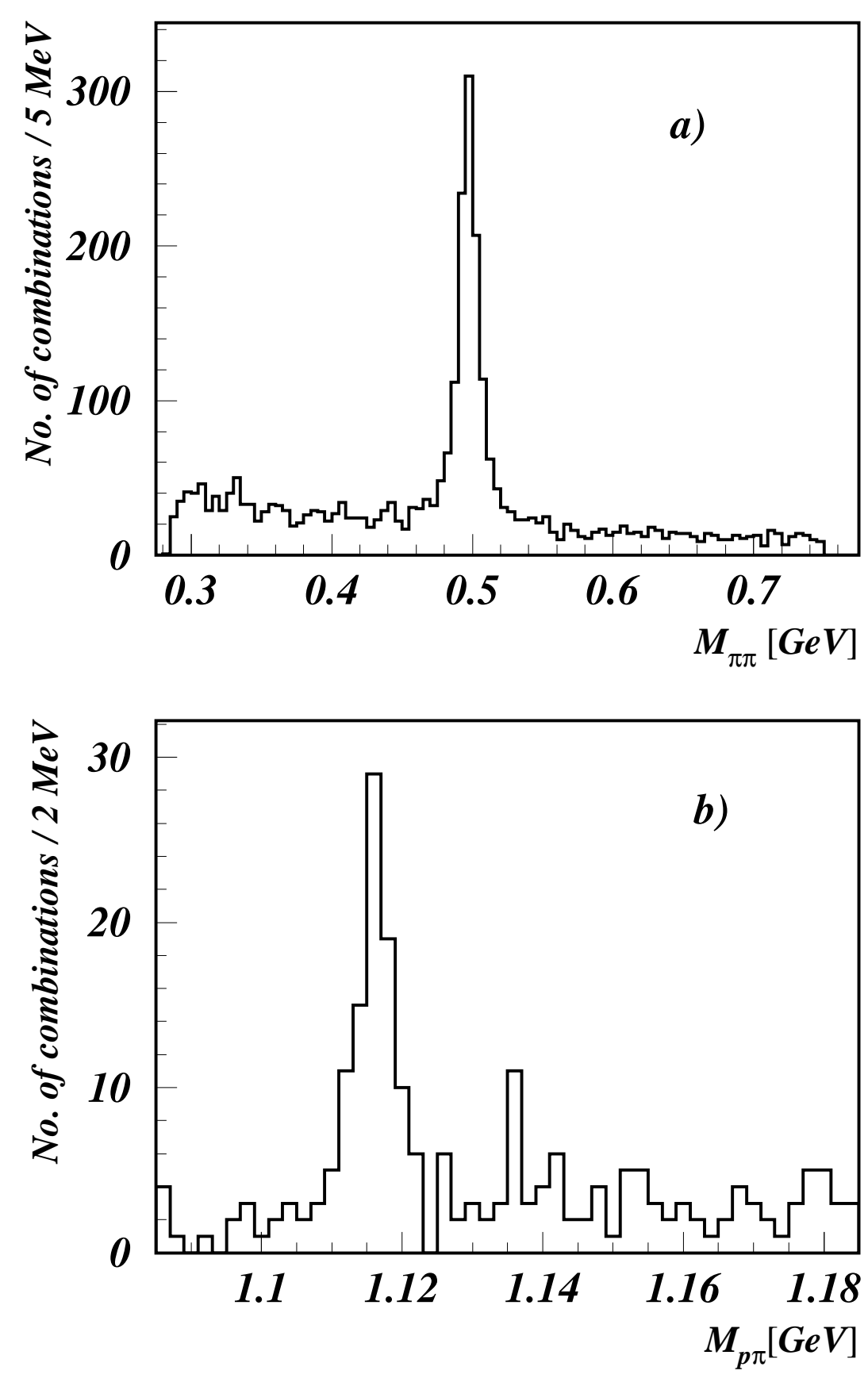

Figure 2: a) Reconstructed $K_{S}^{0}$ mass distribution and b) reconstructed $\Lambda$ mass distribution in accepted events with $10<Q^{2}<640 \mathrm{GeV}^{2}, 0.0003<x<0.01$, and $y>0.04$. The kinematic range of accepted $K_{S}^{0}$ candidates is $0.5<p_{\mathrm{T}}<4 \mathrm{GeV}$ and for $\Lambda$ candidates it is $0.5<p_{\mathrm{T}}<3.5 \mathrm{GeV}$. The $\eta$ range of accepted $K_{S}^{0}$ and $\Lambda$ candidates is $|\eta|<1.3$. 


\section{ZEUS 1993}
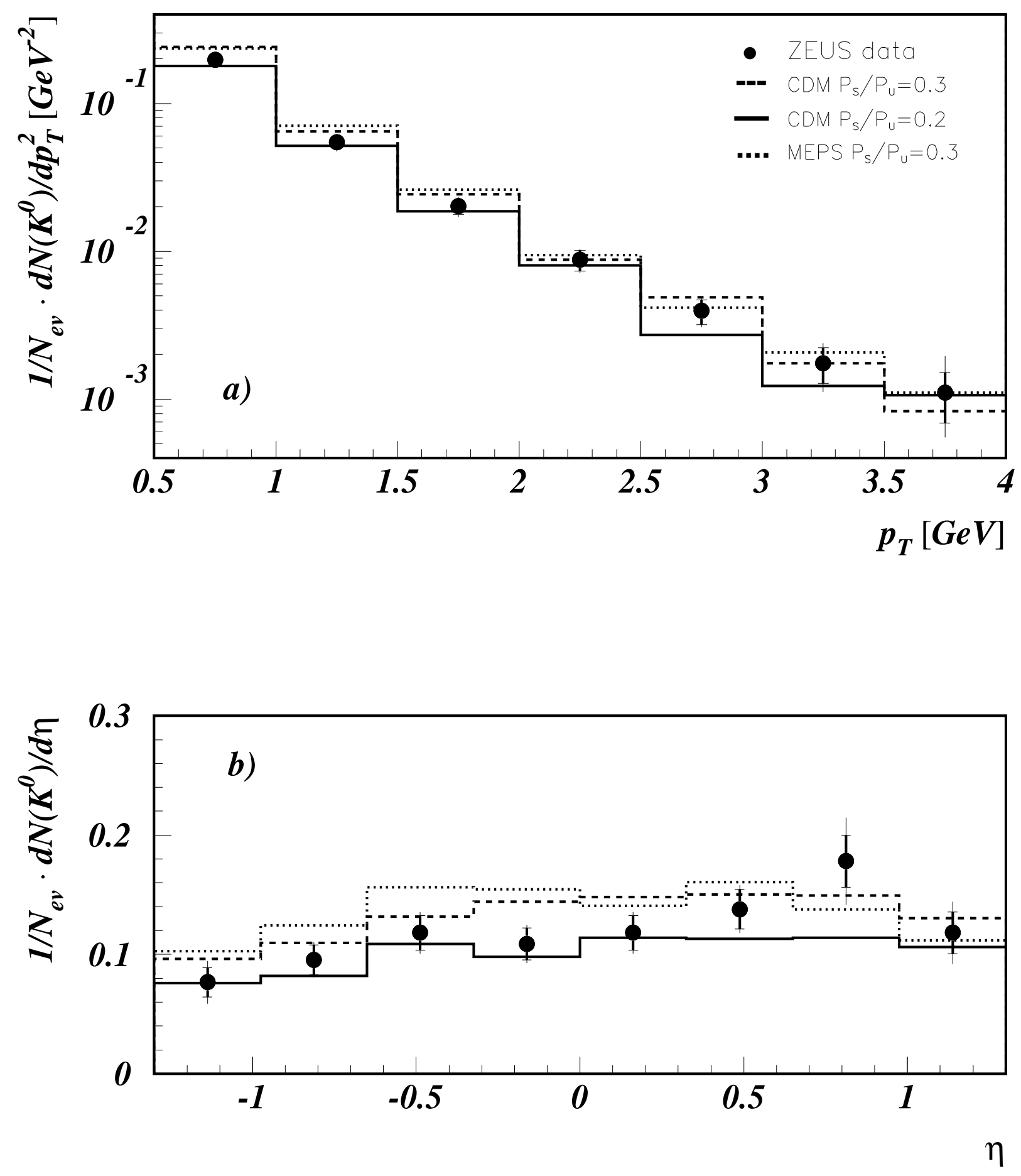

Figure 3: a) Differential multiplicity of $K^{0}$ versus transverse momentum of the kaons in the restricted kinematic $\eta$ and $p_{\mathrm{T}}$ range; b) same for the pseudorapidity of the kaons. The inner error bars show the statistical error and the outer ones correspond to the statistical and systematic errors added in quadrature. The predictions of the three models discussed in the text are shown. 


\section{ZEUS 1993}
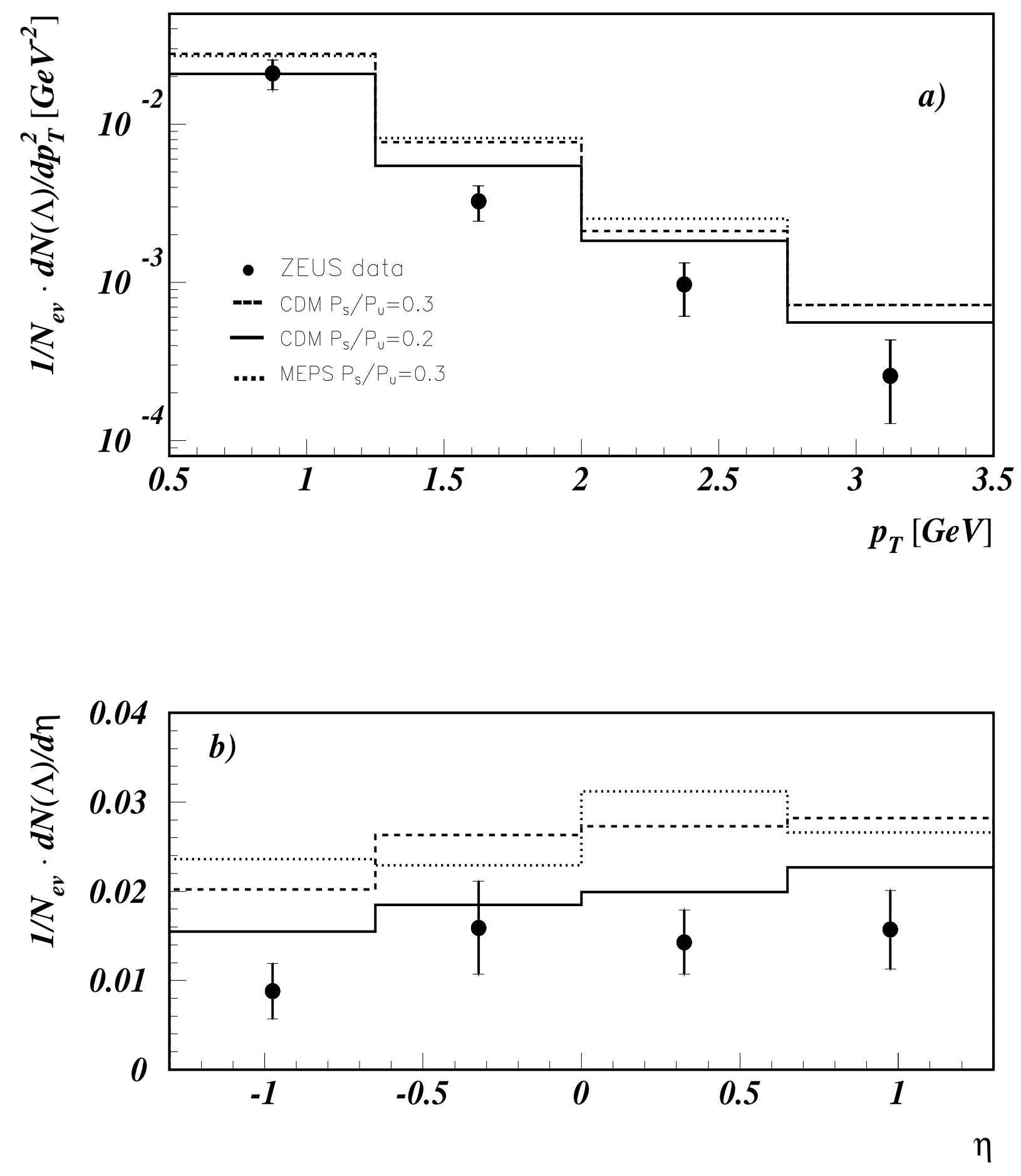

Figure 4: a) Differential multiplicity of $\Lambda$ versus transverse momentum in the restricted kinematic $p_{\mathrm{T}}$ and $\eta$ range; b) same for the pseudorapidity of the $\Lambda$ 's. The inner error bars show the statistical error and the outer ones correspond to the statistical and systematic errors added in quadrature. Statistical errors dominate. The predictions of the three models discussed in the text are shown. 


\section{ZEUS 1993}

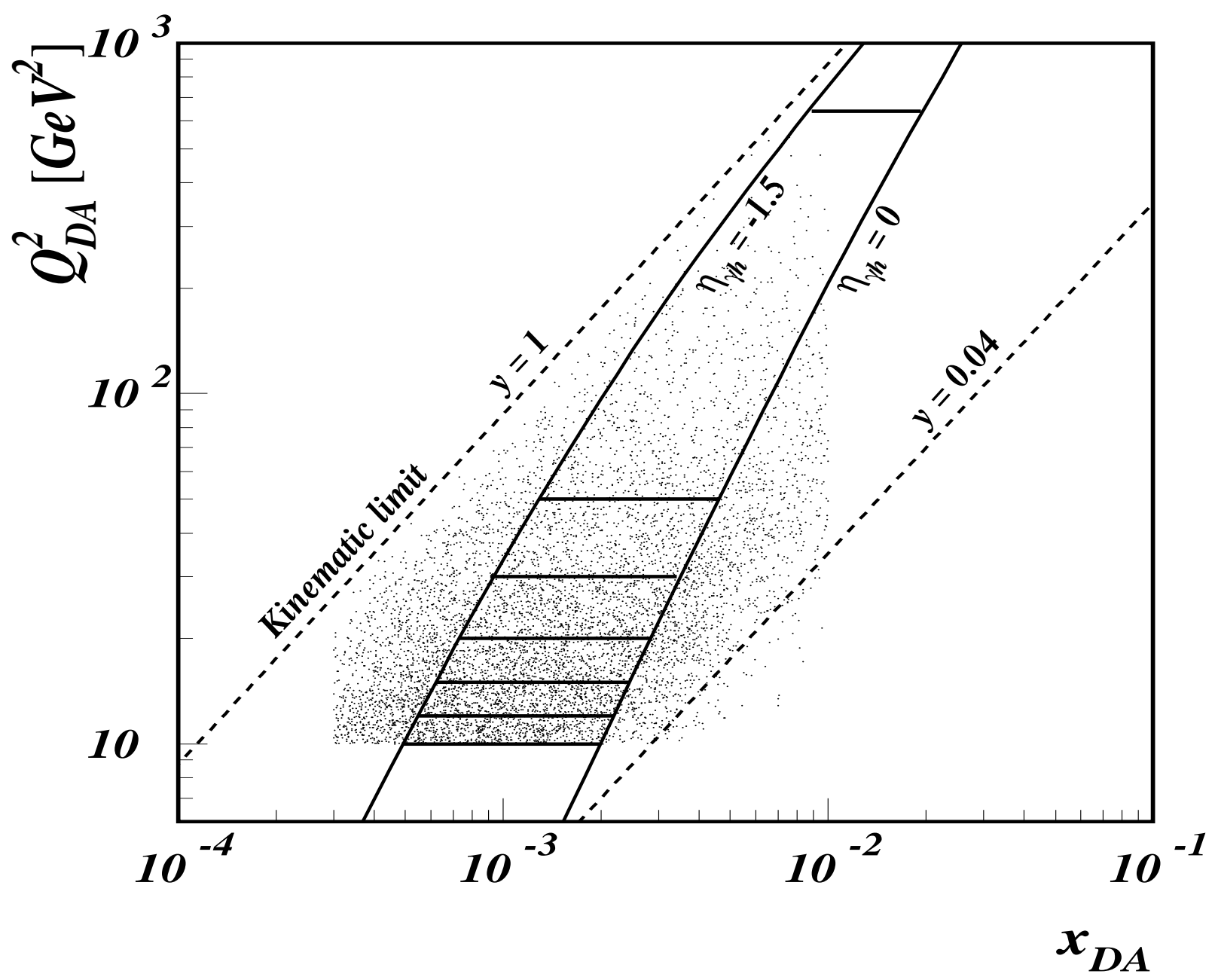

Figure 5: The $\left(x, Q^{2}\right)$ plane with lines of constant $\eta_{\gamma_{h}}$. For the determination of the $Q^{2}$ dependence of the $K^{0}$ multiplicity only events with $-1.5<\eta_{\gamma_{h}}<0$ are accepted. The horizontal lines correspond to the $Q^{2}$ bins chosen. 


\section{ZEUS 1993}
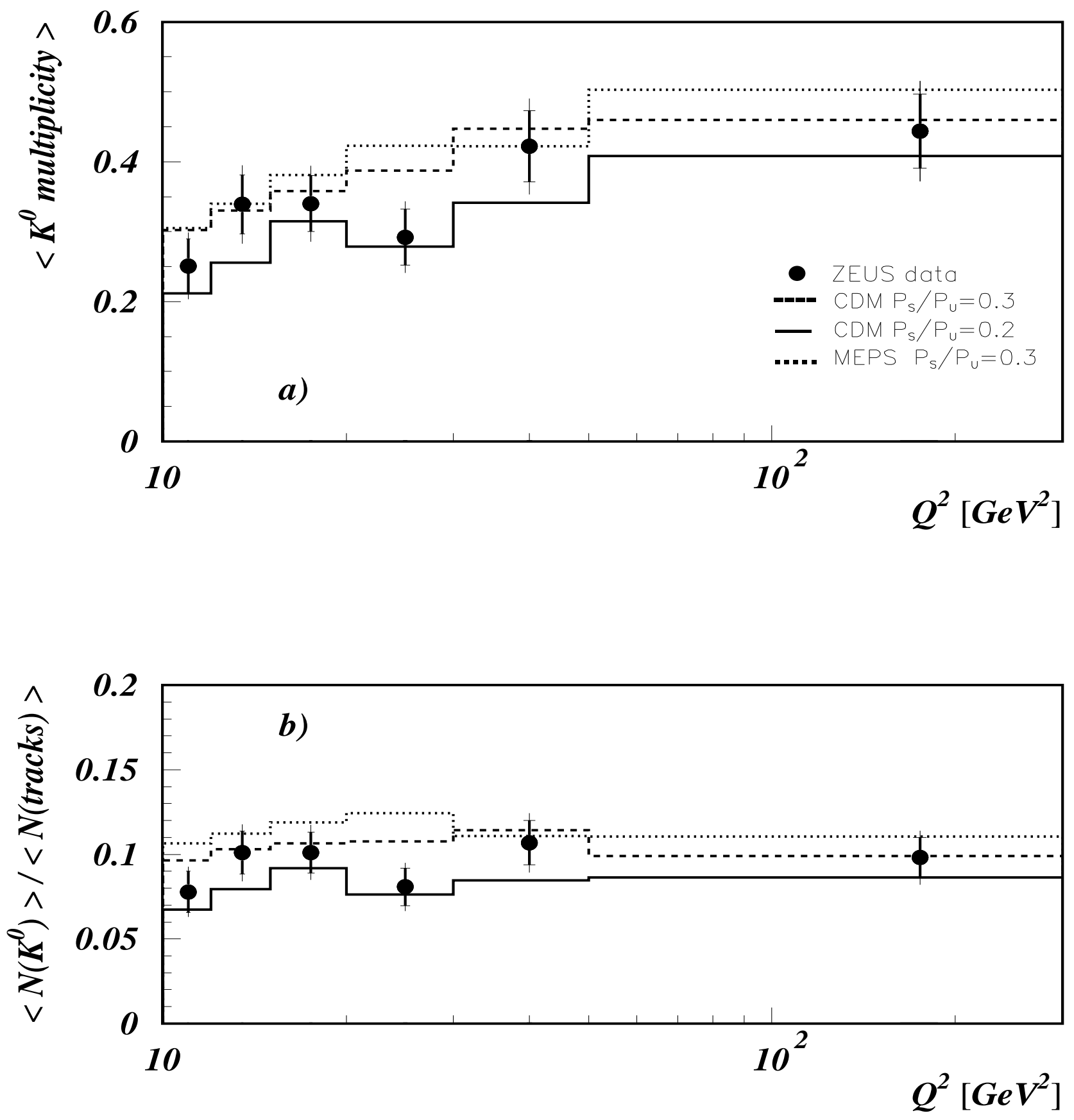

Figure 6: a) Mean multiplicity of $K^{0}$ versus the $Q^{2}$ of the event in the restricted kinematic ranges. b) Ratio of the mean $K^{0}$ multiplicity to the mean charged particle multiplicity as a function of the event's $Q^{2}$. The inner error bars show the statistical error and the outer ones correspond to the statistical and systematic errors added in quadrature. The predictions of three Monte Carlo samples are shown. 


\section{ZEUS 1993}
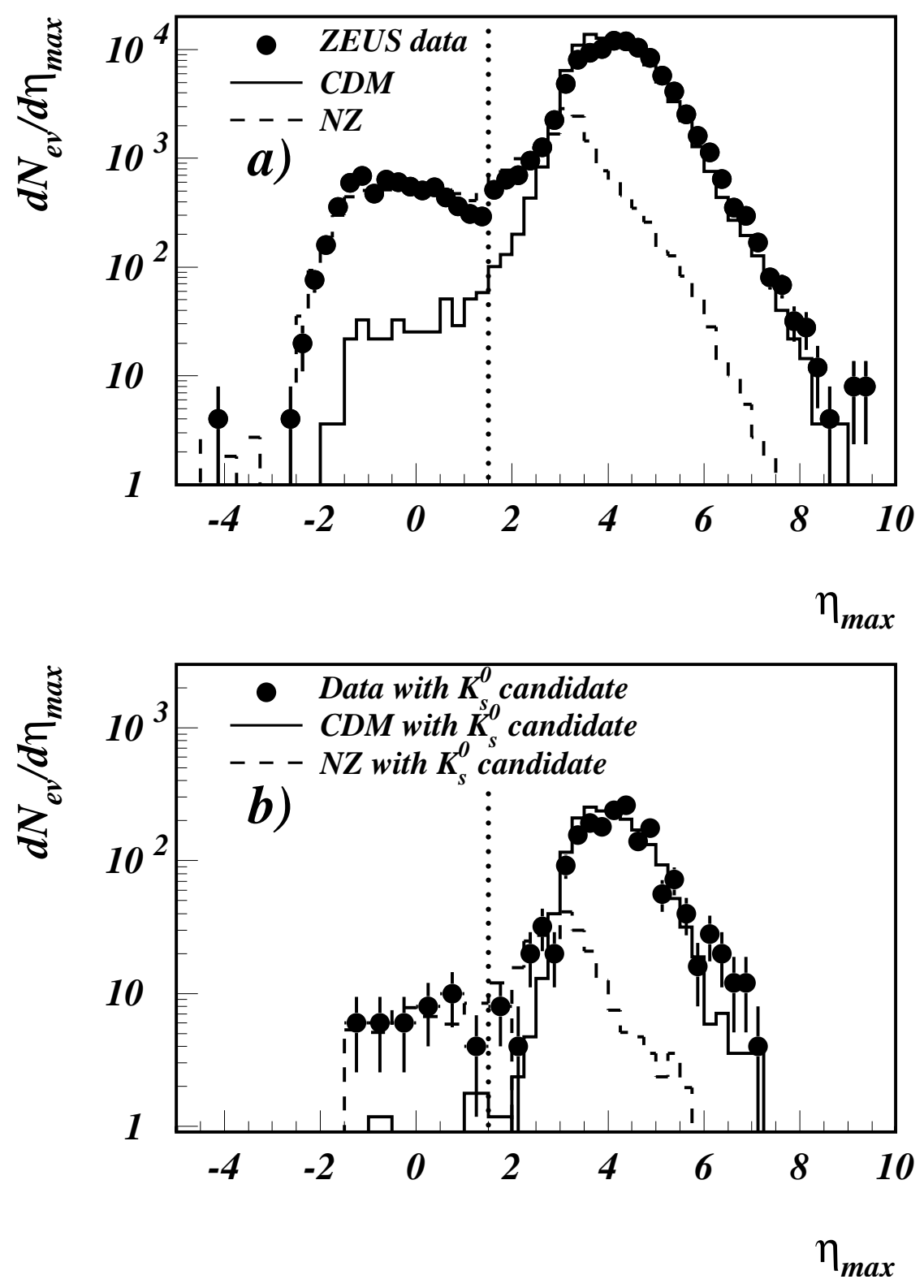

Figure 7: a) $\eta_{\max }$ distribution for all selected events, for one of the non-diffractive Monte Carlo samples (CDM) and for one of the diffractive Monte Carlo samples (NZ). The relative fractions of CDM $(88 \%)$ and NZ(12\%) events are chosen so that their sum reproduces the data distribution best. b) $\eta_{\max }$ distribution of events with a $K_{S}^{0}$ candidate in the signal region of the $M_{\pi \pi}$ distribution. The predictions of the CDM model and of the NZ model are overlaid. The dotted line corresponds to $\eta_{\max }$ $=1.5$. 


\section{ZEUS 1993}
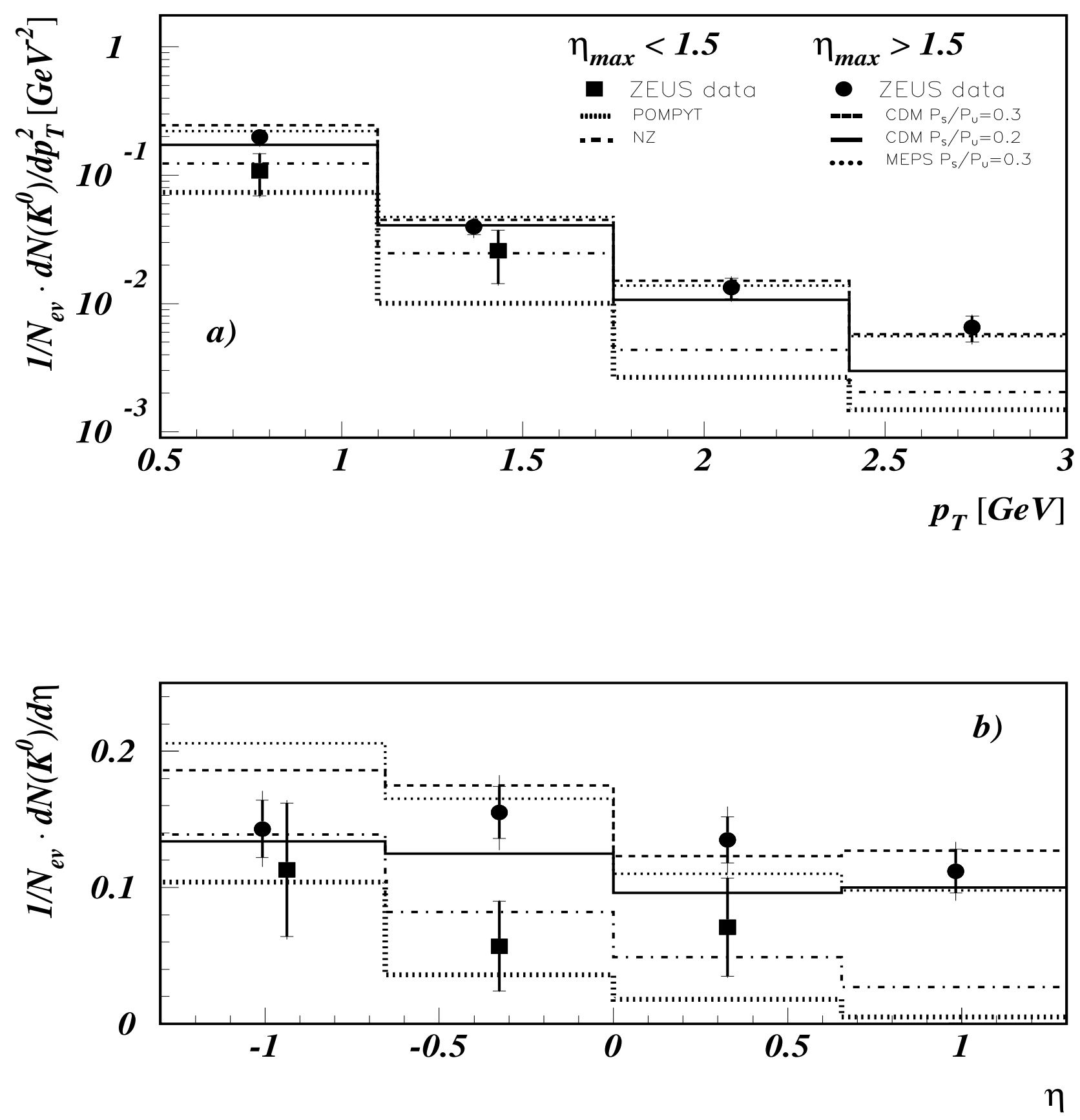

Figure 8: Differential $K^{0}$ multiplicity in NRG DIS and LRG DIS events as a function of their a) transverse momentum; and b) pseudorapidity. The inner error bars show the statistical error and the outer ones correspond to the statistical and systematic errors added in quadrature. The predictions of five Monte Carlo samples are shown. The two diffractive Monte Carlo samples are generated with $P_{s} / P_{u}=0.3$. 